IZA DP No. 6065

Bounds on Average and Quantile Treatment Effects of Job Corps Training on Wages

German Blanco

Carlos A. Flores

Alfonso Flores-Lagunes

October 2011 


\title{
Bounds on Average and Quantile Treatment Effects of Job Corps Training on Wages
}

\author{
German Blanco \\ State University of New York at Binghamton
}

Carlos A. Flores

University of Miami

\section{Alfonso Flores-Lagunes}

State University of New York at Binghamton

and IZA

Discussion Paper No. 6065

October 2011

\author{
IZA \\ P.O. Box 7240 \\ 53072 Bonn \\ Germany \\ Phone: +49-228-3894-0 \\ Fax: +49-228-3894-180 \\ E-mail: iza@iza.org
}

\begin{abstract}
Any opinions expressed here are those of the author(s) and not those of IZA. Research published in this series may include views on policy, but the institute itself takes no institutional policy positions.

The Institute for the Study of Labor (IZA) in Bonn is a local and virtual international research center and a place of communication between science, politics and business. IZA is an independent nonprofit organization supported by Deutsche Post Foundation. The center is associated with the University of Bonn and offers a stimulating research environment through its international network, workshops and conferences, data service, project support, research visits and doctoral program. IZA engages in (i) original and internationally competitive research in all fields of labor economics, (ii) development of policy concepts, and (iii) dissemination of research results and concepts to the interested public.
\end{abstract}

IZA Discussion Papers often represent preliminary work and are circulated to encourage discussion. Citation of such a paper should account for its provisional character. A revised version may be available directly from the author. 
IZA Discussion Paper No. 6065

October 2011

\section{ABSTRACT \\ Bounds on Average and Quantile Treatment Effects of Job Corps Training on Wages*}

We assess the effectiveness of Job Corps (JC), the largest job training program targeting disadvantaged youth in the United States, by constructing nonparametric bounds for the average and quantile treatment effects of the program on wages. Our preferred estimates point toward convincing evidence of positive effects of JC on wages both at the mean and throughout the wage distribution. For the different demographic groups analyzed, the statistically significant estimated average effects are bounded between 4.6 and 12 percent, while the quantile treatment effects are bounded between 2.7 and 11.7 percent. Furthermore, we find that the program's effect on wages varies across quantiles and groups. Blacks likely experience larger impacts in the lower part of their wage distribution, while Whites likely experience larger impacts in the upper part of their distribution. Non-Hispanic Females show statistically significant impacts in the upper part of their distribution but not in the lower part.

JEL Classification: J24, I38, C21

Keywords: $\quad$ training programs, wages, bounds, quantile treatment effects

Corresponding author:

Alfonso Flores-Lagunes

Department of Economics

State University of New York at Binghamton

P.O. Box 6000

Vestal, NY 13902-6000

USA

E-mail: aflores@binghamton.edu

\footnotetext{
* We thank Oscar Mitnik for detailed comments, and participants at the 2011 Institute for Research in Poverty Summer Workshop and at Binghamton University's labor group for useful comments.
} 


\section{Introduction}

Assessment of the effect of government labor market programs on participants' outcomes (e.g., earnings, education, employment) is of great importance to policy makers. To compare these programs' effectiveness to their public cost, one relies on the ability to estimate the causal effects of the program, which is usually a difficult task. The vast majority of both empirical and methodological econometric literature on program evaluation (e.g., Heckman, LaLonde and Smith, 1999; Imbens and Wooldridge, 2009) focuses on estimating causal effects of participation on total earnings, which is a basic step for a cost-benefit analysis. Evaluating the impact on total earnings, however, leaves open a relevant question about whether or not these programs have a positive effect on the wages of participants through the accumulation of human capital, which is an important goal of active labor market programs.

Total earnings are the product of the individual's wage times hours worked. In other words, earnings have two components: price of labor and quantity supplied of labor. By focusing on estimating the impact of program participation on earnings one cannot distinguish how much of the effect is due to human capital improvements. Assessing the labor market effect of program participation on human capital requires focusing on the price component of earnings, i.e., wages. The reason is that wages are directly related to the improvement of participants' human capital through the program. Unfortunately, estimation of the program's effect on wages is not straightforward due to the well-known sample selection problem (Heckman, 1979). Essentially, wages are observed only for those individuals who are employed. Even randomization of program participation does not solve this problem, as the comparison of wages between treatment and control groups does not result in causal effects because the individual's decision to become employed is endogenous and occurs after randomization.

In this paper, we use data from the National Job Corps Study (NJCS), a randomized evaluation of the Job Corps (JC) program, to empirically assess the effect of JC training on wages. We analyze effects both at the mean and at different quantiles of the wage distribution of participants, as well as for different demographic groups. To accomplish this objective, we construct nonparametric bounds that require weaker assumptions than those conventionally employed for point identification of average treatment effects in 
the presence of sample selection $\sqrt[1]{1}$ We focus on estimating bounds on the population of individuals who would be employed regardless of participation in JC, as previously done in Lee (2009) and Zhang et al. (2008), among others. The main reason is that wages are non-missing under both treatment arms for this group of individuals, thus requiring fewer assumptions to construct bounds on their effect. Furthermore, this is an important group of participants: it is estimated to be the largest group among eligible JC participants, accounting for close to 60 percent of them.

Our analysis starts by computing the Horowitz and Manski (2000) "worst-case" bounds, which exploit the randomization in the NJCS and use the empirical support of the outcome. However, these bounds are too wide (i.e., uninformative) in our application. Subsequently, we proceed to tighten the bounds through the use of monotonicity assumptions within a principal stratification framework (Frangakis and Rubin, 2002). We employ two types of monotonicity assumptions. The first type states individual-level weak monotonicity of the effect of the program on employment. This assumption was also employed by Lee (2009) to partially identify average wage effects of JC. The second type of weak monotonicity assumption, which was not considered by Lee (2009), is on mean potential outcomes across strata, which are subpopulations defined by the potential values of the employment status variable under both treatment arms. These assumptions result in informative bounds for the parameters of interest.

We contribute to the literature in several ways. We provide a substantive empirical analysis of the effect of the JC training program on wages. With a yearly cost of about $\$ 1.5$ billion, JC is America's largest job training program. As such, this federally funded program is under constant examination and, given legislation seeking to cut federal spending, the program's operational budget is currently under scrutiny (e.g., USA Today, 2011). Our results provide evidence on the effectiveness of this program in increasing wages. Moreover, they answer a policy-relevant question regarding the potential

\footnotetext{
${ }^{1}$ Many of the methods employed for point identification of average treatment effects under sample selection require strong distributional assumptions that may not be satisfied in practice, such as bivariate normality (Heckman, 1979). One may relax this distributional assumption by relying on exclusion restrictions (Heckman, 1990; Imbens and Angrist, 1994), which require variables that determine selection into the sample (employment) but do not affect the outcome (wages). It is well known, however, that in the case of employment and wages it is difficult to find plausible exclusion restrictions (Angrist and Krueger, 1999; Angrist and Krueger, 2001).
} 
heterogeneity of the wage impacts of JC at different points of the wage distribution, and across different demographic groups. In this way, we complement the original work by Lee (2009) who analyzed the average effect of JC on wages, and contribute to a growing literature analyzing the effectiveness of active labor market programs across different demographic groups (Heckman and Smith, 1999; Abadie, Angrist, and Imbens, 2002; Flores-Lagunes, Gonzalez, and Neumann, 2009; Flores et al., forthcoming). Finally, we illustrate a way to analyze treatment effects on different quantiles of the distribution of an outcome in the presence of sample selection by employing the set of monotonicity assumptions described above $\mathrm{2}^{2}$ In doing so, we provide one of the first applications in economics of recently proposed sharp bounds for quantile treatment effects by Imai $(2008) \cdot 3$

Our results characterize the heterogeneous impact of JC training at different points of the wage distribution. The estimated bounds for a sample that excludes Hispanics strongly suggest positive effects of JC on wages, both at the mean and throughout the wage distribution. For the different demographic groups analyzed, the statistically significant estimated average effects are bounded between 4.6 and 12 percent, while the quantile treatment effects are bounded between 2.7 and 11.7 percent. 4 Our analysis by race and gender reveals that the positive effects for Blacks appear larger in the lower half of their wage distribution, while for Whites the effects appear larger in the upper half of their wage distribution. Lastly, non-Hispanic Females in the lower part of their wage distribution do not show statistically significant positive effects of JC on their wages, while those in the upper part do.

The rest of the paper is organized as follows. Section 2 briefly describes the Job Corps program and the National Job Corps Study data. Section 3 formally presents the sample selection problem and introduces the building block for the identification strategy we employ to bound treatment effects. Section 4 describes the principal stratification framework and the assumptions employed to construct and tighten bounds on average treatment effects. Section 5 discusses bounds on quantile treatment effects. Section 6

\footnotetext{
${ }^{2}$ Other recent work (to be discussed below) that employs bounds on quantile treatment effects under different monotonicity assumptions are Blundell et al. (2007) and Lechner and Melly (2010).

${ }^{3}$ Other models of quantile treatment effects rely on instrumental variables (Abadie, Angrist and Imbens (2002) and Chernozhukov and Hansen (2005)), while the partial identification strategy does not.

${ }^{4}$ The reason why Hispanics are excluded from the analysis is discussed in the next section.
} 
presents the results of our analysis of the Job Corps program. We conclude in section 7.

\section{Job Corps and the National Job Corps Study}

Job Corps (JC) is America's largest and most comprehensive education and job training program. It was established in 1964 as part of the War on Poverty under the Economic Opportunity Act, is federally funded, and is currently administered by the US Department of Labor. With a yearly cost of about $\$ 1.5$ billion, JC annual enrollment ascends to 100,000 students (US Department of Labor, 2010). The program's goal is to help disadvantaged young people, ages 16 to 24, improve the quality of their lives by enhancing their labor market opportunities and educational skills set. Eligible participants receive academic, vocational, and social skills training at over 123 centers nationwide (US Department of Labor, 2010), where they typically reside. Participants are selected based on several criteria, including age, legal US residency, economically disadvantage status, living in a disruptive environment, in need of additional education or training, and be judged to have the capability and aspirations to participate in JC (Schochet et al., 2001).

Being the nation's largest job training program, the effectiveness of JC has been debated at times. During the mid nineties, the US Department of Labor commissioned Mathematica Policy Research, Inc. to design and implement a randomized evaluation, the National Job Corps Study (NJCS), in order to determine the program's effectiveness. The main feature of the study was its random assignment: individuals were taken from nearly all JC's outreach and admissions agencies located in the 48 continuous states and the District of Columbia and randomly assigned to treatment and control groups. During the sample intake period from November 1994 to February 1996, a total of 80,883 first time eligible applicants were included in the study. From this total, approximately 12 percent were assigned to the treatment group $(9,409)$ and 7 percent to the control group $(5,977)$. The remaining 65,497 were assigned to a program non-research group (Schochet et al., 2001). After recording their data through a baseline interview for both treatment and control experimental groups, a series of follow up interviews were conducted at weeks 52, 130, and 208 after randomization.

Randomization took place before participants' assignment to a JC center. As a result, only 73 percent of the individuals randomly assigned to the treatment group actually 
enrolled in JC. Also, about 1.4 percent of the individuals assigned to the control group enrolled in the program despite the three-year embargo imposed on them (Schochet et al., 2001). Therefore, in the presence of this non-compliance, the comparison of outcomes by random assignment to the treatment has the interpretation of the "intention-to-treat" (ITT) effect, that is, the causal effect of being offered participation in JC. Focusing on this parameter in the presence of non-compliance is common practice in the literature (e.g., Lee, 2009; Flores-Lagunes et al., 2009; Zhang et al., 2009). Correspondingly, our empirical analysis focuses on estimating informative non-parametric bounds for ITT effects, although for simplicity we describe our methods and results in the context of treatment effects.

We start our analysis with the same sample employed by Lee (2009), who developed an intuitive trimming procedure for bounding the average treatment effect of JC on participants' wages. This sample is restricted to individuals who have non-missing values for weekly earnings and weekly hours for every week after random assignment, resulting in a sample size of $9,1455^{5}$ We use this sample to compare our results to Lee (2009) and to analyze the informational content of our additional assumption to tighten the estimated bounds. Subsequently, we restrict the sample by excluding Hispanics, which renders a sample size of 7,573 . The reason to drop Hispanics is that, in contrast to all other demographic groups in the NJCS sample, it has been documented that this group exhibited negative (albeit not statistically significant) impacts of JC on both employment and earnings (e.g., Schochet et al., 2001; Flores-Lagunes et al., 2009). Since one of our main assumptions is individual-level monotonicity of the effect of JC on employment (to be discussed in section 4), we prefer to leave this group out of the remaining analysis because inclusion of this group would likely violate this assumption ${ }^{6}$ Finally, due to both programmatic and design reasons in the NJCS, different subgroups in the study population had different probabilities of being included in the research sample. Thus,

\footnotetext{
${ }^{5}$ As a consequence, we implicitly assume - as do the studies cited in the previous paragraph - that the missing values are "missing completely at random". For a recent study employing a (parametric) likelihood-based analysis to account for non-compliance, missing observations, and sample selection, see Frumento et al. (2010).

${ }^{6}$ Nevertheless, we obtained a full set of results for the sample of Hispanics. Accordingly, most of the estimated bounds were uninformative, and in some instances they could not be computed due to a strong failure of the individual-level monotonicity assumption.
} 
throughout our analysis, we employ the NJCS design weights (Schochet, 2001) 7

Summary statistics for the sample of 9,145 individuals, which essentially replicate those of Lee (2009, p. 1075), are presented in the Internet Appendix. Pretreatment variables in the data include: demographic variables, education and background variables, income variables, and employment information. As expected, given the randomization, the distribution of these pretreatment characteristics is similar across treatment and control groups, with the difference in the means of both groups being not statistically significant at a 5 percent level. The resulting difference for post-treatment earnings in week 208 after random assignment across groups in this sample is quantitatively equivalent and consistent with the previously found 12 percent positive effect of JC on participants' weekly earnings (Burghardt et al., 1999; Schochet et al., 2001). Results on the effect of JC on participants' weekly hours worked in this sample of about two hours a week are also consistent with those obtained in previous studies (Schochet et al., 2001). Similar summary statistics for the groups to be analyzed (Non-Hispanics, Blacks, Whites, Non-Hispanic Males, and Non-Hispanic Females) are also relegated to the Internet Appendix.

\section{The Sample Selection Problem and the Horowitz and Manski Bounds}

Assessing the impact of job training programs on wages is fundamentally distinct than assessing the program's impact on earnings. Earnings are the product of the individual's wage times hours worked, therefore, the impact on earnings encompasses the effect on the likelihood of being employed (labor supply effect) and the effect on wages. Thus, the impact on wages can be interpreted as a pure price effect since significant increases in wages can be directly related to the improvement of the participants' human capital due to the program, which is essential for individuals to boost their labor market opportunities. Indeed, one of JC's main goals is the enhancement of participants' human capital through academic and vocational training. Thus, it is of considerable importance to evaluate the program's impact on wages.

\footnotetext{
${ }^{7}$ For example, outreach and admissions agencies had struggle recruiting females for residential slots in the past. Therefore, sampling rates to the control group were intentionally set lower for females in some areas to overcome potential difficulties with unfilled slots.
} 
Estimation of a program's causal effect on wages is complicated - even in the presence of random assignment - by the fact that only the wages of those employed are observed. This is referred to in the literature as the sample selection problem (Heckman, 1979). Formally, consider having access to data on $N$ individuals and define a binary treatment $T_{i}$, which indicates whether individual $i$ has participated in the program $\left(T_{i}=1\right)$ or not $\left(T_{i}=0\right)$. We start with an assumption that accords with our data:

Assumption A. $T_{i}$ is randomly assigned.

To illustrate the sample selection problem, assume for the moment that the individual's wage is a linear function of a constant term, the treatment indicator $T_{i}$ and a set of pretreatment characteristics $X_{1 i}:^{8}$

$$
Y_{i}^{*}=\beta_{0}+T_{i} \beta_{1}+X_{1 i} \beta_{2}+U_{1 i}
$$

where $Y_{i}^{*}$ is the latent wage for individual $i$, which is observed conditional on the selfselection process into employment. This process is also assumed (for the moment) to be linearly related to a constant, the treatment indicator $T_{i}$ and a set of pretreatment characteristics $X_{2 i}$,

$$
S_{i}^{*}=\delta_{0}+T_{i} \delta_{1}+X_{2 i} \delta_{2}+U_{2 i}
$$

Similarly, $S_{i}^{*}$ is a latent variable representing the individual's propensity to be employed. Let $S_{i}$ denote the observed employment indicator that takes values $S_{i}=1$ if individual $i$ is employed and 0 otherwise. Then,

$$
S_{i}=1\left[S_{i}^{*} \geq 0\right]
$$

where 1[.] is an indicator function. Therefore, we observe individual $i$ 's wage, $Y_{i}$, when $i$ is employed $\left(S_{i}=1\right)$ and it remains latent when unemployed $\left(S_{i}=0\right)$.

Conventionally, point identification of the parameter of interest, $\beta_{1}$ (assumed to be constant over the population in this setting), requires strong assumptions such as joint independence of the errors $\left(U_{1 i}, U_{2 i}\right)$ in the wage and employment equations (1) and (2) and the regressors $T_{i}, X_{1 i}$ and $X_{2 i}$, plus bivariate normality of $\left(U_{1 i}, U_{2 i}\right)$ (Heckman, 1979). The bivariate normality assumption about the errors can be relaxed by relying on

\footnotetext{
${ }^{8}$ Linearity is assumed here to simplify the exposition of the sample selection problem. The nonparametric approach to address sample selection employed in this paper does not impose linearity or functional form assumptions to partially identify the treatment effects of interest.
} 
exclusion restrictions (Heckman, 1990; Heckman and Smith, 1995; Imbens and Angrist, 1994), which require variables that determine employment but do not affect wages, or equivalently, variables in $X_{2 i}$ that do not belong in $X_{1 i}$. However, it is well known that finding such variables that go along with economic reasoning in this situation is extremely difficult (Angrist and Krueger, 1999; Angrist and Krueger, 2001).

An alternative approach suggests that the parameters can be bounded without relying on distributional assumptions or on the availability and validity of exclusion restrictions. Horowitz and Manski (2000; HM hereafter) proposed a general framework to construct bounds on treatment effects when data is missing due to a nonrandom process, such as self-selection into non-employment $\left(S_{i}^{*}<0\right)$, provided that the outcome variable has a bounded support.9 These bounds are known in the literature as "worst-case" bounds.

To illustrate HM's bounds, let $Y_{i}(0)$ and $Y_{i}(1)$ be the potential (counterfactual) wages for unit $i$ under control $\left(T_{i}=0\right)$ and treatment $\left(T_{i}=1\right)$, respectively. The relationship between these potential wages and the observed $Y_{i}$ is that $Y_{i}=Y_{i}(1) T_{i}+Y_{i}(0)\left(1-T_{i}\right)$. Define the average treatment effect $(A T E)$ as

$$
A T E=E\left[Y_{i}(1)-Y_{i}(0)\right]=E\left[Y_{i}(1)\right]-E\left[Y_{i}(0)\right] .
$$

Conditional on $T_{i}$ and the observed employment indicator $S_{i}$, the $A T E$ in (3) can be written as:

$$
\begin{aligned}
A T E & =E\left[Y_{i} \mid T_{i}=1, S_{i}=1\right] \operatorname{Pr}\left(S_{i}=1 \mid T_{i}=1\right) \\
& +E\left[Y_{i}(1) \mid T_{i}=1, S_{i}=0\right] \operatorname{Pr}\left(S_{i}=0 \mid T_{i}=1\right) \\
& -E\left[Y_{i} \mid T_{i}=0, S_{i}=1\right] \operatorname{Pr}\left(S_{i}=1 \mid T_{i}=0\right) \\
& -E\left[Y_{i}(0) \mid T_{i}=0, S_{i}=0\right] \operatorname{Pr}\left(S_{i}=0 \mid T_{i}=0\right)
\end{aligned}
$$

Examination of Equation (4) reveals that, under random assignment, we can identify from the data all the conditional probabilities $\left(\operatorname{Pr}\left(S_{i}=s \mid T_{i}=t\right)\right.$, for $\left.(t, s)=(0,1)\right)$ and also the expectations of the wage when conditioning on $S_{i}=1\left(E\left[Y_{i} \mid T_{i}=1, S_{i}=1\right]\right.$ and $\left.E\left[Y_{i} \mid T_{i}=0, S_{i}=1\right]\right)$. Unfortunately, sample selection into non-employment makes it impossible to point identify $E\left[Y_{i}(1) \mid T_{i}=1, S_{i}=0\right]$ and $E\left[Y_{i}(0) \mid T_{i}=0, S_{i}=0\right]$. We can, however, construct HM bounds on these unobserved objects provided that the support of the outcome lies in a bounded interval $\left[Y^{L B}, Y^{U B}\right]$, since this implies that the values

\footnotetext{
${ }^{9}$ Horowitz and Manski (2000) derived conservative bounds on parameters of interest using nonparametric analysis applied to experimental settings with problems of missing binary outcomes and covariates.
} 
for these unobserved objects are restricted to such interval. Thus, HM's lower and upper bounds ( $L B^{H M}$ and $U B^{H M}$, respectively) are identified as follows:

$$
\begin{aligned}
L B^{H M} & =E\left[Y_{i} \mid T_{i}=1, S_{i}=1\right] \operatorname{Pr}\left(S_{i}=1 \mid T_{i}=1\right)+Y^{L B} \operatorname{Pr}\left(S_{i}=0 \mid T_{i}=1\right) \\
& -E\left[Y_{i} \mid T_{i}=0, S_{i}=1\right] \operatorname{Pr}\left(S_{i}=1 \mid T_{i}=0\right)-Y^{U B} \operatorname{Pr}\left(S_{i}=0 \mid T_{i}=0\right) \\
U B^{H M} & =E\left[Y_{i} \mid T_{i}=1, S_{i}=1\right] \operatorname{Pr}\left(S_{i}=1 \mid T_{i}=1\right)+Y^{U B} \operatorname{Pr}\left(S_{i}=0 \mid T_{i}=1\right) \\
& -E\left[Y_{i} \mid T_{i}=0, S_{i}=1\right] \operatorname{Pr}\left(S_{i}=1 \mid T_{i}=0\right)-Y^{L B} \operatorname{Pr}\left(S_{i}=0 \mid T_{i}=0\right)
\end{aligned}
$$

Note that these bounds do not employ distributional or exclusion restrictions assumptions. They are nonparametric and allow for heterogeneous treatment effects, that is, non-constant effects over the population. On the other hand, a cost of disposing of those assumptions is that the HM bounds are often uninformative. Indeed, this is the case in our application as will be shown below. For this reason, we take this approach as a building block and proceed by imposing more structure through the use of assumptions that are typically weaker than the distributional and exclusion restriction assumptions needed for point identification.

\section{Bounds on Average Treatment Effects}

We follow the approach by Lee (2009) and Zhang et al. (2008) who employ monotonicity assumptions that lead to a trimming procedure that tightens the HM bounds. They implicitly or explicitly employ the principal stratification framework of Frangakis and Rubin (2002) to motivate and derive their results. Principal stratification provides a framework for analyzing average causal effects when controlling for a post-treatment variable that has been affected by treatment assignment. In the context of the effect of JC on wages, the affected post-treatment variable is employment. In this framework, individuals are classified into "principal strata" based on the potential values of employment under each treatment arm. Comparisons of outcomes by treatment assignment within strata can be interpreted as causal effects because which strata an individual belongs to is not affected by treatment assignment.

More formally, let the potential values of employment be denoted by $S_{i}(0)$ and $S_{i}(1)$ when $i$ is assigned to control and treatment, respectively. We can partition the population into strata based on the values of the vector $\left\{S_{i}(0), S_{i}(1)\right\}$. Since both $S_{i}$ and $T_{i}$ are 
binary, there are four principal strata:

$$
\begin{aligned}
& N N:\left\{S_{i}(0)=0, S_{i}(1)=0\right\} \\
& E E:\left\{S_{i}(0)=1, S_{i}(1)=1\right\} \\
& E N:\left\{S_{i}(0)=1, S_{i}(1)=0\right\} \\
& N E:\left\{S_{i}(0)=0, S_{i}(1)=1\right\} .
\end{aligned}
$$

In the context of $\mathrm{JC}, N N$ is the stratum of those individuals who would be unemployed regardless of treatment assignment, while $E E$ is the stratum of those who would be employed regardless of treatment assignment. The stratum $E N$ represents those who would be employed if assigned to control but unemployed if assigned to treatment, and $N E$ is the stratum of those who would be unemployed if assigned to control but employed if assigned to treatment. Given that strata are defined based on the potential values of $S_{i}$, the stratum an individual belongs to is unobserved. A mapping of the observed groups based on $\left(T_{i}, S_{i}\right)$ to the unobserved strata above is depicted in the first two columns of Table 1.

Lee (2009) and Zhang et al. (2008) focus on the average treatment effect of a program on wages for individuals who would be employed regardless of treatment status, i.e., the $E E$ stratum. This stratum is the only one for which wages are observed under both treatment arms, and thus fewer assumptions are required to construct bounds for its effects. We focus on the effects on this same stratum. Thus, the average treatment effect parameter we concentrate on is:

$$
A T E_{E E}=E\left[Y_{i}(1) \mid E E\right]-E\left[Y_{i}(0) \mid E E\right] .
$$

\subsection{Bounds Adding an Individual-Level Monotonicity Assump- tion}

To tighten the HM bounds presented in Section 3, we can employ the following individual-level monotonicity assumption about the relationship between the treatment (JC) and employment:

Assumption B. Individual-Level Positive Weak Monotonicity of $T$ on $S(T)$ : $S_{i}(1) \geq$ $S_{i}(0)$ for all $i$.

This assumption states that treatment assignment affects employment (weakly) in one direction, effectively ruling out the EN stratum. Both Lee (2009) and Zhang et al. (2008) 
employed this assumption. In the context of JC, Assumption B is plausible because one of the program's stated goals is to increase the employability of participants. It does so by providing academic, vocational and social skills training to participants, as well as job search assistance. Indeed, the NJCS reported a positive and highly statistically significant average effect of JC on employment (Schochet et al. 2001).

Nevertheless, this assumption can be criticized since it assumes the sign of the individual treatment effect of the program on employment (e.g., Lechner and Melly, 2010) ${ }^{10}$ Two factors that may cast doubt on this assumption in our setting are that individuals are "locked-in" away from employment while undergoing training (van Ours, 2004), and the possibility that trained individuals may have a higher reservation wage after training and thus may choose to remain unemployed (e.g., Blundell et al., 2007). Note, however, that these two factors become less relevant the longer the time horizon after randomization at which the outcome is measured. For this reason, we focus on wages at the $208^{\text {th }}$ week after random assignment, which is the latest wage measure available in the NJCS.11 In addition, there is one demographic group in our sample for which Assumption B is likely not appropriate. Hispanics in the NJCS were the only group found to have negative but statistically insignificant effects of JC on both earnings and employment (Schochet et al., 2001; Flores-Lagunes et al., 2009). Thus, in the main analysis to be presented below, we consider a sample that excludes this group. Lastly, Assumption B can be falsified by the data, as it gives rise to the following testable implication (Zhang et al., 2008; Imai, 2008): $\operatorname{Pr}(S=0 \mid T=0)-\operatorname{Pr}(S=0 \mid T=1) \geq 0$. We employ this testable implication in our empirical analysis below.

Assumption B, by virtue of eliminating the EN stratum, allows the identification of some individuals in the $E E$ and $N N$ strata, as can be seen after deleting the $E N$ stratum in the last column of Table 1. Furthermore, the combination of Assumptions A and $\mathrm{B}$ point identifies the proportions of each principal strata in the population. Let $\pi_{k}$ be the population proportions of each principal strata $k=N N, E E, E N, N E$, and let $p_{S \mid T} \equiv \operatorname{Pr}\left(S_{i}=s \mid T_{i}=t\right)$ for $t, s=0,1$. Then, $\pi_{E E}=p_{1 \mid 0}, \pi_{N N}=p_{0 \mid 1}, \pi_{N E}=p_{1 \mid 1}-p_{1 \mid 0}=$

\footnotetext{
${ }^{10}$ Lechner and Melly (2010) relax this individual-level monotonicity by making it hold conditional on observed covariates.

${ }^{11}$ Zhang et al. (2009) provide some evidence that the estimated proportion of individuals who do not satisfy the individual-level assumption (the $E N$ stratum) falls with the time horizon at which the outcome is measured after randomization.
} 
$p_{0 \mid 0}-p_{0 \mid 1}$ and $\pi_{E N}=0$. Looking at the last column of Table 1, we know that individuals in the observed group with $\left(T_{i}, S_{i}\right)=(0,1)$ belong to the stratum of interest $E E$. Therefore, we can point identify $E\left[Y_{i}(0) \mid E E\right]$ in (7) with $E\left[Y_{i} \mid T_{i}=0, S_{i}=1\right]$. However, it is not possible to point identify $E\left[Y_{i}(1) \mid E E\right]$, since the observed group with $\left(T_{i}, S_{i}\right)=(1,1)$ is a mixture of individuals from two strata, $E E$ and $N E$. Nevertheless, it can be bounded. We can write $E\left[Y_{i} \mid T_{i}=1, S_{i}=1\right]$ as a weighted average of individuals belonging to the $E E$ and $N E$ strata:

$$
E\left[Y_{i} \mid T_{i}=1, S_{i}=1\right]=\frac{\pi_{E E}}{\left(\pi_{E E}+\pi_{N E}\right)} E\left[Y_{i}(1) \mid E E\right]+\frac{\pi_{N E}}{\left(\pi_{E E}+\pi_{N E}\right)} E\left[Y_{i}(1) \mid N E\right]
$$

Since the proportion of $E E$ individuals in the group $\left(T_{i}, S_{i}\right)=(1,1)$ can be point identified as $\pi_{E E} /\left(\pi_{E E}+\pi_{N E}\right)=p_{1 \mid 0} / p_{1 \mid 1}, E\left[Y_{i}(1) \mid E E\right]$ can be bounded from above by the expected value of $Y_{i}$ for the $\left(p_{1 \mid 0} / p_{1 \mid 1}\right)$ fraction of the largest values of $Y_{i}$ in the observed group $\left(T_{i}, S_{i}\right)=(1,1)$. In other words, the upper bound is obtained under the scenario that the largest $\left(p_{1 \mid 0} / p_{1 \mid 1}\right)$ values of $Y_{i}$ belong to the $E E$ individuals. Thus, computing the expected value of $Y_{i}$ after trimming the lower tail of the distribution of $Y_{i}$ in $\left(T_{i}, S_{i}\right)=(1$, 1 ) by $1-\left(p_{1 \mid 0} / p_{1 \mid 1}\right)$ yields an upper bound for the $E E$ group. Similarly, $E\left[Y_{i}(1) \mid E E\right]$ can be bounded from below by the expected value of $Y_{i}$ for the $\left(p_{1 \mid 0} / p_{1 \mid 1}\right)$ fraction of the smallest values of $Y_{i}$ for those in the same observed group. The resulting upper $\left(U B_{E E}\right)$ and lower $\left(L B_{E E}\right)$ bounds for $A T E_{E E}$ are (Lee, 2009; Zhang et al., 2008):

$$
\begin{gathered}
U B_{E E}=E\left[Y_{i} \mid T_{i}=1, S_{i}=1, Y_{i} \geq y_{1-\left(p_{1 \mid 0} / p_{1 \mid 1}\right)}^{11}\right]-E\left[Y_{i} \mid T_{i}=0, S_{i}=1\right] \\
L B_{E E}=E\left[Y_{i} \mid T_{i}=1, S_{i}=1, Y_{i} \leq y_{\left(p_{1 \mid 0} / p_{1 \mid 1}\right)}^{11}\right]-E\left[Y_{i} \mid T_{i}=0, S_{i}=1\right],
\end{gathered}
$$

where $y_{1-\left(p_{1 \mid 0} / p_{1 \mid 1}\right)}^{11}$ and $y_{\left(p_{1 \mid 0} / p_{1 \mid 1}\right)}^{11}$ denote the $1-\left(p_{1 \mid 0} / p_{1 \mid 1}\right)$ and the $\left(p_{1 \mid 0} / p_{1 \mid 1}\right)$ quantiles of $Y_{i}$ conditional on $T_{i}=1$ and $S_{i}=1$, respectively. Lee (2009) shows that these bounds are sharp (i.e., there are no shorter bounds possible under the current assumptions).

To estimate the bounds in (9) we can simply substitute sample quantities for population quantities:

$$
\begin{aligned}
\widehat{U B_{E E}} & =\frac{\sum_{i=1}^{n} Y_{i} \cdot T_{i} \cdot S_{i} \cdot 1\left[Y_{i} \geq \widehat{y_{1-\hat{p}}}\right]}{\sum_{i=1}^{n} T_{i} \cdot S_{i} \cdot 1\left[Y_{i} \geq \widehat{y_{1-\hat{p}}}\right]}-\frac{\sum_{i=1}^{n} Y_{i} \cdot\left(1-T_{i}\right) \cdot S_{i}}{\sum_{i=1}^{n}\left(1-T_{i}\right) \cdot S_{i}} \\
\widehat{L B_{E E}} & =\frac{\sum_{i=1}^{n} Y_{i} \cdot T_{i} \cdot S_{i} \cdot 1\left[Y_{i} \leq \widehat{y_{\hat{p}}}\right]}{\sum_{i=1}^{n} T_{i} \cdot S_{i} \cdot 1\left[Y_{i} \leq \widehat{y_{\hat{p}}}\right]}-\frac{\sum_{i=1}^{n} Y_{i} \cdot\left(1-T_{i}\right) \cdot S_{i}}{\sum_{i=1}^{n}\left(1-T_{i}\right) \cdot S_{i}}
\end{aligned}
$$

where $\widehat{y_{1-\hat{p}}}$ and $\widehat{y_{\hat{p}}}$ are the sample analogs of the quantities $y_{1-\left(p_{1 \mid 0} / p_{1 \mid 1}\right)}^{11}$ and $y_{\left(p_{1 \mid 0} / p_{1 \mid 1}\right)}^{11}$ in (9), respectively, and $\hat{p}$, the sample analog of $\left(p_{1 \mid 0} / p_{1 \mid 1}\right)$, is calculated as follows:

$$
\hat{p}=\frac{\sum_{i=1}^{n}\left(1-T_{i}\right) \cdot S_{i}}{\sum_{i=1}^{n}\left(1-T_{i}\right)} / \frac{\sum_{i=1}^{n} T_{i} \cdot S_{i}}{\sum_{i=1}^{n} T_{i}}
$$


Lee (2009) shows that these estimators are asymptotically normal and employs them to estimate the average effect of JC on wages at different time horizons after randomization. Below, we will replicate his results for wages at week 208 after randomization. We will also obtain corresponding estimates for relevant groups and estimate alternative tighter bounds that impose more structure that we argue is plausible in the current setting.

\subsection{Adding Weak Monotonicity of Mean Potential Outcomes Across Strata to Tighten the Bounds}

We present a weak monotonicity assumption of mean potential outcomes across the $E E$ and $N E$ strata level that tightens the bounds in (9). This assumption was originally proposed by Zhang and Rubin (2003) and employed in Zhang et al. (2008):

Assumption C. Weak Monotonicity of Mean Potential Outcomes Across the EE and $N E$ Strata: $E[Y(1) \mid E E] \geq E[Y(1) \mid N E]$.

Intuitively, this assumption formalizes the notion that the $E E$ stratum is likely to be comprised of more "able" individuals than those belonging to the $N E$ stratum. Since "ability" is positively correlated with labor market outcomes (e.g., wages and employment), one would expect wages for the individuals who are employed regardless of treatment status (the EE stratum) to weakly dominate on average the wages of those individuals who are employed only if they receive training (the $N E$ stratum). While Assumption $\mathrm{C}$ is not directly testable, one can indirectly gauge its plausibility by comparing the average of pre-treatment variables that are highly correlated with wages between the $E E$ and $N E$ strata. ${ }^{12}$ We illustrate this in our analysis below. Assumption $\mathrm{C}$ is related to-but different from-Manski and Pepper's (2000) "monotone instrumental variable" assumption. Their assumption states that mean responses vary weakly monotonically across subpopulations defined by specific values of the instrument. In contrast, Assumption C compares mean responses across two principal strata.

Employing Assumptions A, B, and C results in tighter bounds. To see this, recall

\footnotetext{
${ }^{12}$ In a setting where the outcome is not truncated due to non-employment, Flores and Flores-Lagunes (2010) show that Assumption C provides testable implications that can be employed to falsify it. Unfortunately, in our current setting, the unobservability of wages for those unemployed prevents the computation of these testable implications.
} 
that the average outcome in the observed group with $\left(T_{i}, S_{i}\right)=(1,1)$ contains units from two strata, $E E$ and $N E$, and can be written as the weighted average shown in (8). By replacing $E\left[Y_{i}(1) \mid N E\right]$ with $E\left[Y_{i}(1) \mid E E\right]$ in 8 and using the inequality in Assumption $\mathrm{C}$, we have that $E\left[Y_{i} \mid T_{i}=1, S_{i}=1\right] \leq E\left[Y_{i}(1) \mid E E\right]$, and thus that $E\left[Y_{i}(1) \mid E E\right]$ is bounded from below by $E\left[Y_{i} \mid T_{i}=1, S_{i}=1\right]$. Therefore, the lower bound for $A T E_{E E}$ becomes: $E\left[Y_{i} \mid T_{i}=1, S_{i}=1\right]-E\left[Y_{i} \mid T_{i}=0, S_{i}=1\right]$. Imai (2008) shows that these bounds are sharp.

To estimate the bounds under Assumptions A, B, and C, note that the upper bound estimate of 9 remains $\widehat{U B_{E E}}$ from 10 , while the estimate for the lower bound is the corresponding sample analog of $E\left[Y_{i} \mid T_{i}=1, S_{i}=1\right]-E\left[Y_{i} \mid T_{i}=0, S_{i}=1\right]$ :

$$
\widehat{L B_{E E}^{c}}=\frac{\sum_{i=1}^{n} Y_{i} \cdot T_{i} \cdot S_{i}}{\sum_{i=1}^{n} T_{i} \cdot S_{i}}-\frac{\sum_{i=1}^{n} Y_{i} \cdot\left(1-T_{i}\right) \cdot S_{i}}{\sum_{i=1}^{n}\left(1-T_{i}\right) \cdot S_{i}} .
$$

\section{Bounds on Quantile Treatment Effects}

We now extend the results presented in the previous section to the construction of bounds on quantile treatment effects $(Q T E)$ based on results by Imai (2008). The parameters of interest are defined as the difference in the quantiles of the distributions of the potential outcomes $Y(1)$ and $Y(0)$. This difference is well-defined as long as the marginal distributions of potential outcomes are point or partially identified. Our parameter of interest is the $\alpha$-quantile effect for the $E E$ stratum:

$$
Q T E_{E E}^{\alpha}=F_{Y_{i}(1) \mid E E}^{-1}(\alpha)-F_{Y_{i}(0) \mid E E}^{-1}(\alpha)
$$

where $F_{Y_{i}(t) \mid E E}^{-1}(\alpha)$ denotes the $\alpha$ quantile of the distribution of $Y_{i}(t)$ for the $E E$ stratum.

Two recent papers have focused on partial identification of QTE. Blundell, et. al., (2007) derived sharp bounds on the distribution of wages and the interquantile range to study income inequality in the U.K. Their work builds on the worst-case bounds on the conditional quantiles in Manski (1994), which are tighten by imposing stochastic dominance assumptions. Their stochastic dominance assumption is applied to the distribution of wages of individuals observed employed and unemployed, whereby the wages of employed individuals are assumed to weakly dominate those of unemployed individuals (i.e., positive selection into employment). In addition, they explore the use of exclusion restrictions to further tighten their bounds. Lechner and Melly (2010) analyze QTE of 
a German training program on wages. They impose an individual-level monotonicity assumption similar to our Assumption B that is weakened by conditioning on covariates $X$, and they subsequently employ the stochastic dominance assumption of Blundell et al. (2007) to tighten their bounds. In contrast to those papers, we take advantage of the randomization in the NJCS to estimate QTE by employing individual-level monotonicity (Assumption B) and by strengthening Assumption C to stochastic dominance applied to the $E E$ and $N E$ strata. Another difference between those studies and ours is the parameters of interest. While Blundell et al. (2007) focus on the population QTE, Lechner and Melly (2010) focus on the QTE for those individuals who are employed under treatment. Our focus is on the QTE for individuals who are employed regardless of treatment assignment (the $E E$ stratum) 13

Let $F_{Y_{i} \mid T_{i}=t, S_{i}=s}(\cdot)$ be the cumulative distribution of individuals' wages conditional on $T_{i}=t$ and $S_{i}=s$, and let $y_{\alpha}^{t s}$ denote its corresponding $\alpha$-quantile, for $\alpha \in(0,1)$, or $y_{\alpha}^{t s}=$ $F_{Y_{i} \mid T_{i}=t, S_{i}=s}^{-1}(\alpha)$. Following the same intuition for partial identification of $E\left[Y_{i}(1) \mid E E\right]$ by trimming the observed quantity $E\left[Y_{i} \mid T_{i}=1, S_{i}=1\right]$, we can partially identify $Q T E_{E E}^{\alpha}$ as follows:

Proposition 1 (Imai, 2008). Under assumptions $A$ and $B, L B_{E E}^{\alpha} \leq Q T E_{E E}^{\alpha} \leq U B_{E E}^{\alpha}$, where

$$
\begin{aligned}
U B_{E E}^{\alpha} & =F_{Y_{i} \mid T_{i}=1, S_{i}=1, Y_{i} \geq y_{1-\left(p_{1 \mid 0} / p_{1 \mid 1}\right)}^{11}}^{-1}(\alpha) \\
& -F_{Y_{i} \mid T_{i}=0, S_{i}=1}^{-1}(\alpha) \\
L B_{E E}^{\alpha} & =F_{Y_{i} \mid T_{i}=1, S_{i}=1, Y_{i} \leq y_{\left(p_{1 \mid 0} / p_{1 \mid 1}\right)}^{11}}^{-1}(\alpha) \\
& -F_{Y_{i} \mid T_{i}=0, S_{i}=1}^{-1}(\alpha)
\end{aligned}
$$

Similar to $(9), F_{Y_{i} \mid T_{i}=1, S_{i}=1, Y_{i} \geq y_{1-\left(p_{1 \mid 0} / p_{1 \mid 1}\right)}^{11}}(\cdot)$ and $F_{Y_{i} \mid T_{i}=1, S_{i}=1, Y_{i} \leq y_{\left(p_{1 \mid 0}^{11} / p_{1 \mid 1}\right)}^{11}}(\cdot)$ correspond to the upper and lower bounding distributions of the wages of those individuals who belong to $E E$ in the observed group $\left(T_{i}, S_{i}\right)=(1,1)$. As such, $U B_{E E}^{\alpha}$ is an upper bound for the difference in quantiles between the treated and control groups' outcomes at a given $\alpha$-quantile for the $E E$ stratum. Similarly, $L B_{E E}^{\alpha}$ represents a lower bound for this difference. Imai (2008) shows that the bounds in (14) are sharp.

\footnotetext{
${ }^{13}$ The treated-and-employed subpopulation is a mixture of two strata: $E E$ and $N E$. In our application, the $E E$ stratum and the treated-and-employed subpopulation account for about the same proportion of the population (57 and 61 percent, respectively).
} 
We estimate the bounds in (14) as:

$$
\begin{aligned}
& \widehat{U B_{E E}^{\alpha}}=\widehat{y_{\alpha}^{u}}-\widehat{y_{\alpha}^{c}} \\
& \widehat{L B_{E E}^{\alpha}}=\widehat{y_{\alpha}^{l}}-\widehat{y_{\alpha}^{c}},
\end{aligned}
$$

where the $\alpha$-quantile for each marginal distribution is calculated as:

$$
\widehat{y_{\alpha}^{h}}=\min \left\{y: \frac{\sum_{i=1}^{n} T_{i} \cdot S_{i} \cdot 1\left[Y_{i}^{h} \leq y\right]}{\sum_{i=1}^{n} T_{i} \cdot S_{i}} \geq \alpha\right\},
$$

with $h=\{u, l\}$ for the upper and lower bounding distribution, respectively, and $Y_{i}^{h}$ representing the outcome of individuals in the group with $\left[T_{i}=1, S_{i}=1, Y_{i} \geq y_{1-\left(p_{1 \mid 0} / p_{1 \mid 1}\right)}^{11}\right]$ for $h=u$ or $\left[T_{i}=1, S_{i}=1, Y_{i} \leq y_{\left(p_{1 \mid 0} / p_{1 \mid 1}\right)}^{11}\right]$ for $h=l$. Similarly, the $\alpha$-quantile for the observed control group with $\left(T_{i}, S_{i}\right)=(0,1)$ is calculated as:

$$
\widehat{y_{\alpha}^{c}}=\min \left\{y: \frac{\sum_{i=1}^{n}\left(1-T_{i}\right) \cdot S_{i} \cdot 1\left[Y_{i}^{c} \leq y\right]}{\sum_{i=1}^{n}\left(1-T_{i}\right) \cdot S_{i}} \geq \alpha\right\}
$$

with $Y_{i}^{c}$ representing the outcome of the individuals in the group with $\left(T_{i}, S_{i}\right)=(0,1)$.

\subsection{Tightening Bounds on QTE using Stochastic Dominance}

We tighten the bounds in (14) by employing an assumption similar to Assumption C in section 4.2. For the case of $Q T E$, this assumption has to be strengthened to stochastic dominance:

Assumption D. Stochastic Dominance Between the EE and NE Strata:: $F_{Y_{i}(1) \mid E E}(y) \leq$ $F_{Y_{i}(1) \mid N E}(y)$,

where $F_{Y_{i} \mid E E}(\cdot)$ and $F_{Y_{i} \mid N E}(\cdot)$ are the cumulative distributions of wages for individuals who belong to the $E E$ and $N E$ strata, respectively.

This assumption directly imposes restrictions on the distribution of potential outcomes under treatment for individuals in the $E E$ stratum, which results in a tighter lower bound relative to that in (14). After adding this assumption, the resulting sharp bounds are:

Proposition 2 (Imai, 2008). Under assumptions $A, B$, and $D, L B_{E E}^{d \alpha} \leq Q T E_{E E}^{\alpha} \leq$ $U B_{E E}^{\alpha}$, where $U B_{E E}^{\alpha}$ is as in (14) and

$$
L B_{E E}^{d \alpha}=F_{Y_{i} \mid T_{i}=1, S_{i}=1}^{-1}(\alpha)-F_{Y_{i} \mid T_{i}=0, S_{i}=1}^{-1}(\alpha)
$$

The estimator of the upper bound is still given by $\widehat{U B_{E E}^{\alpha}}$ in $(15)$, while the estimator for $L B_{E E}^{d \alpha}$ is now given by:

$$
\widehat{L B_{E E}^{d \alpha}}=\widehat{y_{\alpha}^{l}}-\widehat{y_{\alpha}^{c}}
$$


where $\widehat{y_{\alpha}^{l}}=\min \left\{y: \frac{\sum_{i=1}^{n} T_{i} \cdot S_{i} \cdot 1\left[Y_{i}^{t} \leq y\right]}{\sum_{i=1}^{n} T_{i} \cdot S_{i}} \geq \alpha\right\}$, and $Y_{i}^{t}$ represents the outcome of those individuals in the group with $\left(T_{i}, S_{i}\right)=(1,1)$.

\section{Estimation of Bounds on the Effect of Job Corps on Wages}

In this section we empirically assess the effect of JC training on wages using data from the NJCS. In Section 6.1, we concentrate on the average treatment effect and compute the HM bounds under random assignment (Assumption A). Subsequently, we estimate bounds for $A T E_{E E}$ that add different assumptions in order to tighten these benchmark bounds. Section 6.2 reports bounds derived under Assumptions A and individual-level monotonicity (Assumption B), while Section 6.3 explores the identifying power of weak monotonicity of mean potential outcomes across strata (Assumption C). Sections 6.4 and 6.5 present and discuss bounds on $Q T E_{E E}^{\alpha}$.

\subsection{Horowitz and Manski (HM) bounds}

Table 2 reports the HM bounds, which only employ random assignment (Assumption A), for the average treatment effect of JC on log wages in week 208 after randomization. The table shows two sets of bounds. In the first, we follow Lee (2009) and transform $\log$ wages to minimize the effect of outliers on the width of these bounds by splitting the entire observed wage distribution into 20 percentile groups $\left(5^{t h}, 10^{\text {th }}, \ldots\right.$, and $95^{\text {th }}$ percentile of log wages) and assigning to individuals in each percentile the mean log wage in that group. The last column computes the HM bounds using the untransformed log wages to exploit the original variation in this variable and to be able to use these bounds as benchmark when adding other assumptions and when computing bounds on the QTE.

Table 2 shows that Lee's transformed log wages have an upper bound on their support, denoted by $Y^{U B}$ in (5), of 2.77, and a lower bound, $Y^{L B}$, of 0.90 . As expected, the "smoothing" of wages has a large impact on the support of the outcome, since the last column shows that for the untransformed wages the upper and lower bounds on their support are 5.99 and -1.55 , respectively. Consequently, the width of the HM bounds for the untransformed log wages (6.244) is considerably larger than that for the transformed 
ones (1.548). Detailed calculations of all quantities needed to construct the bounds in (5) are shown in the second column of Table 2. Despite the large differences between the two measures of wages, the evidence in Table 2 has the same qualitative implication about the HM bounds: they are largely uninformative. The estimated HM bounds on the average treatment effect of JC on wages using transformed log wages are 0.802 (upper bound) and -0.746 (lower bound), while using untransformed log wages are 3.135 (upper bound) and -3.109 (lower bound). These bounds are the basis upon which we add assumptions to tighten them.

\subsection{Bounds Adding Individual-Level Monotonicity}

Under individual-level monotonicity of JC on employment (Assumption B) we partially identify the average effect of JC on wages for those individuals who are employed regardless of treatment assignment (the $E E$ stratum). Therefore, it is of interest to estimate the size of that stratum relative to the full population, which can be done under Assumptions A and B. Table 3 reports the estimated strata proportions for the full sample (labeled "All") and for demographic groups of interest. The EE stratum accounts for close to 57 percent of the population, making it the largest stratum. The second largest stratum is the "never employed" or $N N$, accounting for 39 percent of the population. Lastly, the $N E$ stratum accounts for 4 percent (the stratum $E N$ is ruled out by Assumption B). The relative magnitudes of the strata largely hold for all demographic groups. Interestingly, Whites have the highest proportion of $E E$ individuals at 66 percent, while Blacks have the lowest at 51 percent.

Table 4 reports estimated bounds for $A T E_{E E}$ for the full sample using (10) under Assumptions A and B, for both transformed and untransformed wages. The second column exactly replicates the results by Lee (2009) using transformed log wages. Relative to the HM bounds, these bounds are much tighter: their width goes from 1.548 in the HM bounds to 0.112. However, the bounds still include zero, as does the Imbens and Manski (2004; IM hereafter) confidence intervals reported in the last row. These confidence intervals include the true parameter of interest with a 95 percent probability. The fourth column of Table 4 reports estimated bounds for $A T E_{E E}$ under Assumptions A and B using the untransformed log wages. Unlike the HM bounds, the present bounding procedure does not depend on the empirical support of the outcome, thereby the effect 
of transforming wages is negligible. While the width of the bounds using either measure of wages is similar, both the bounds and the IM confidence intervals include zero. Thus, from Table 4 we see that Assumption B greatly tightens the HM bounds, although not enough to rule out zero or a small negative effect of JC on wages at week 208.

As discussed in Section 4.1, the untestable individual-level weak monotonicity assumption of the effect of JC on employment may be inadequate in certain circumstances. In the context of JC, the group of Hispanics has been found to be unusual in the sense that the NJCS calculated negative but statistically insignificant average effects of the program on both their employment and weekly earnings at week 208, while for the other groups these effects were positive and highly statistically significant (Schochet et al., 2001).14 This evidence casts doubt on the validity of Assumption B for the group of Hispanics. Therefore, we consider a sample that excludes this group (labelled "Non-Hispanics"), which includes 7,573 individuals ${ }^{15}$

Panel A of Table 5 presents estimated bounds under Assumptions A and B for different demographic groups, along with their width and 95 percent IM confidence intervals. The second column reproduces the bounds in Table 4 for the full sample (All). The third column presents the corresponding estimated bounds for the Non-Hispanics sample. The upper bound for this group is larger than the one for All, while the lower bound is less negative, which is consistent with the discussion above regarding Hispanics. The IM confidence intervals are wider for the non-Hispanics sample relative to All, but they are more concentrated on the positive side of the real line. In terms of the other groups (Whites, Blacks, and Non-Hispanic Males and Females), none of the estimated bounds exclude zero, although Whites and Non-Hispanic Males have a lower bound almost right at zero. In general, the IM confidence intervals for the last four demographic groups are wider than those of the All and Non-Hispanics groups, which is likely a consequence of their smaller sample sizes.

We now check the testable implication of Assumption B mentioned in Section 4.1: $\operatorname{Pr}(S=0 \mid T=0)-\operatorname{Pr}(S=0 \mid T=1) \geq 0$. Note that the left-hand-side of this expression

\footnotetext{
${ }^{14}$ The NJCS reported that Hispanics participating in JC had a statistically insignificant decrease in the probability of employment of 3.1 percentage points (Schochet et al., 2001).

${ }^{15}$ In principle, it is possible to construct bounds on the average effect on the $E E$ stratum that dispose of the individual-level monotonicity assumption (Zhang et al., 2008). However, these bounds are typically too wide, which is the case in our application.
} 
is the proportion of individuals in the $N E$ stratum $\left(\pi_{N E}\right)$, which is reported in Table 3 for all groups except Hispanics. From the table it can be seen that all estimated $N E$ stratum proportions are between 0.04 and 0.06 , and they are statistically significant at a 1 percent level (not shown in the table). For Hispanics, the corresponding proportion is a statistically insignificant 0.0021. This evidence indicates that Assumption B is not falsified by the data for all groups reported in Table 3, and suggests that such evidence is dubious for Hispanics.

We close this section by arguing, as does Lee (2009), that small and negative estimated lower bounds on the effect of JC on wages under the current assumptions can be interpreted as pointing toward positive effects. The reason is that the lower bound is obtained by placing individuals in the $E E$ stratum at the bottom of the distribution of the observed group with $\left(T_{i}, S_{i}\right)=(1,1)$. While this mathematically identifies a valid lower bound, it implies a perfect negative correlation between employment and wages that is implausible from the standpoint of standard models of labor supply, in which individuals with higher predicted wages are more likely to be employed. Indeed, one interpretation that can be given to Assumption C (employed in the next section) is that of formalizing this theoretical notion to tighten the lower bound.

\subsection{Bounds Adding Weak Monotonicity of Mean Potential Out- comes Across Strata}

The columns labelled "A, B, and C" in Table 4 present the estimated bounds for the full sample adding Assumption C, for both transformed and untransformed log wages. This assumption has considerable identifying power as it results in much tighter bounds for the $A T E_{E E}$ compared to the previously estimated bounds, with the width being cut in about half for both measures of log wages. Importantly, employing Assumption $\mathrm{C}$ yields estimated bounds that are informative about the sign of the effect of JC training on log wages at week 208. Bounds on the transformed log wages are 0.034 to 0.093 , and those on the untransformed log wages are 0.037 to 0.099 , with both sets ruling out negative effects. When computing IM confidence intervals on the bounds adding Assumption C, we see in the last row of Table 4 that, with 95 percent confidence, both measures of log wages exclude zero, indicating statistically significantly positive effects of JC. Thus, focusing on the untransformed log wages, the effect of JC for $E E$ individuals is significantly positive 
and falls between 3.7 and 9.9 percent.

Given the strong identifying power of Assumption C, it is important to gauge its plausibility in this application. A direct statistical test is not feasible since the assumption is untestable. However, we indirectly gauge its plausibility by looking at one of its implications. Assumption $\mathrm{C}$ formalizes the idea that the $E E$ stratum possesses traits that result in better labor market outcomes relative to individuals in the $N E$ stratum. Thus, we look at pre-treatment covariates that are highly correlated with log wages at week 208 and test whether, on average, individuals in the $E E$ stratum indeed exhibit better characteristics at baseline relative to individuals in the $N E$ stratum. We focus mainly on the following pre-treatment variables: earnings, whether the individual held a job, months employed (all three in the year prior to randomization), and education at randomization.

To implement this idea, we compute average pre-treatment characteristics for the $E E$ and $N E$ strata. Computing average characteristics for the $E E$ stratum is straightforward since, under Assumptions $\mathrm{A}$ and $\mathrm{B}$, the individuals in the observed group $\left(T_{i}, S_{i}\right)=(0,1)$ belong to and are representative of this stratum. To estimate average characteristics for the $N E$ stratum, note that their average can be written as a function of the averages of the whole population and the other strata, all of which can be estimated under Assumptions $\mathrm{A}$ and $\mathrm{B}$. Let $W$ be a pre-treatment characteristic of interest, then,

$$
E[W \mid N E]=\left\{E[W]-\pi_{E E} E[W \mid E E]-\pi_{N N} E[W \mid N N]\right\} / \pi_{N E} .
$$

The estimated differences between the average pre-treatment variables employed for this exercise for the $E E$ and $N E$ strata were all positive, indicating "better" pre-treatment labor market characteristics for the $E E$ stratum. Formal tests of statistical significance for these differences, however, did not reject their equality (mainly because of the high variance in the estimation of $E[W \mid N E])$. We conclude that this exercise does not provide evidence against Assumption C, while the estimated differences suggest that it is a plausible assumption ${ }^{16}$

Panel B of Table 5 presents the estimated bounds, width, and IM confidence intervals for all groups under Assumptions A, B, and C. The second and third columns show inference for the full population (All) and Non-Hispanics. Although the two sets of

\footnotetext{
${ }^{16}$ The tables corresponding to this exercise can be found in the Internet Appendix. Employing other pre-treatment variables provided similar results (i.e., no evidence against Assumption C).
} 
bounds are of similar width, the bounds for Non-Hispanics are shifted higher to an effect of $\mathrm{JC}$ on wages between 5 to 11.8 percent. In fact, the IM confidence intervals show that, despite the smaller sample size, this average effect is statistically significant with 95 percent confidence.

The estimated bounds for the other demographic groups in Table 5 (panel B) show some interesting results. All of the bounds and IM confidence intervals exclude zero, with the smallest lower bound being that of All at 3.7 percent (all others are 4.6 percent and higher). Remarkably, the estimated bounds for all the demographic groups that exclude Hispanics are relatively similar, suggesting that their average effect of JC on wages for the $E E$ stratum is between about 5 and 12 percent. The differences in the confidence intervals across groups is likely driven by the differences in sample sizes. These results imply unequivocal positive effects of JC on wages across the different demographic groups, and they reinforce the previous notion of a strong identifying power of Assumption C.

\subsection{Bounds on Quantile Treatment Effects Under Random As- signment and Individual-Level Monotonicity}

We proceed to analyze the effects of JC on participant's wages beyond the average impact by providing estimated bounds for quantile treatment effects $(Q T E)$ for the $E E$ stratum, $Q T E_{E E}^{\alpha}$. We start by estimating bounds under Assumptions A and B in this subsection. To summarize the evidence from the computation of QTE at several quantiles, we provide a series of figures for the different groups under analysis ${ }_{17}^{17}$ We concentrate on the log of the untransformed wages for brevity and to fully exploit the original variation in this variable. The estimated QTE under Assumptions $\mathrm{A}$ and $\mathrm{B}$, along with their corresponding IM confidence intervals, are shown in Figure 1.

Recall that the estimated bounds for the $A T E_{E E}$ under the same assumptions presented in Section 6.2 did not rule out zero for any of the groups under analysis. Looking at the estimated bounds on the QTE for the full sample in Figure 1(a), they rule out zero for all lower quantiles up to 0.7. Once IM confidence intervals are computed, though, only the bounds for the 0.2 quantile imply statistically significant positive effects of JC on log wages with 95 percent confidence. Given the argument that Assumption B is likely not satisfied for Hispanics, we look at the group of Non-Hispanics in Figure 1(b). Consistent

\footnotetext{
${ }^{17}$ The complete numerical results are shown in the Internet Appendix
} 
with the results from bounds on average effects, the estimated bounds on $Q T E_{E E}^{\alpha}$ for this group are generally shifted towards the positive space. For this group, the estimated bounds also exclude zero for all lower quantiles up to 0.7 , and the 95 percent IM confidence intervals rule out zero for the 0.5 quantile. The estimated bounds for these two samples suggest that JC is more likely to have positive effects on log wages for the lower quantiles of the wage distribution.

Looking at the results by race, Figures $1(\mathrm{c})$ and $1(\mathrm{~d})$ show that, once again, the estimated bounds for the $Q T E$ exclude zero for a number of lower quantiles up to 0.75 (with the exception of the 0.05 quantile for Whites and the 0.75 quantile for Blacks). However, probably due to the smaller sample sizes, when looking at the 95 percent IM confidence intervals for these groups only quantiles 0.55 and 0.65 for Whites and the 0.05 quantile for Blacks are statistically significant. It is worth noting that these two figures suggest that Blacks may experience more positive effects of $\mathrm{JC}$ on wages in the lower quantiles of the wage distribution, while Whites may experience more positive effects at the upper quantiles. The large width of the IM confidence intervals, however, prevents us from being conclusive about this point.

Figures 1(e) and 1(f) show the corresponding estimated bounds and 95 percent IM confidence intervals for Non-Hispanic Males and Females, respectively. The bounds reflect a trend of excluding zero at the lower quantiles that is similar to that of the previous groups, albeit less clear for Non-Hispanic Females. Interestingly, Non-Hispanic Males show a greater number of estimated bounds excluding zero, which is probably due to a lower degree of heterogeneity in this group relative to Non-Hispanic Females ${ }^{18}$ Looking at the IM confidence intervals, none of them exclude zero for Non-Hispanic Females, while they do for quantiles $0.05,0.1$, and 0.45 for Non-Hispanic Males. These results suggest that inference for Non-Hispanic Females is more difficult due to their greater heterogeneity and smaller sample size.

To end this subsection, we remark that, while the bounds and IM confidence intervals for the average treatment effect of JC on wages under Assumptions A and B were inconclusive about its sign, the analysis of QTE reveals that positive effects of JC on

\footnotetext{
${ }^{18}$ By greater heterogeneity of Non-Hispanic Females relative to Non-Hispanic Males we mean that the former group shows higher standard deviation in key variables such as age, marital and cohabitation status, separated, presence of a child, number of children, and education. This is also true for the average characteristics of the corresponding subset of individuals in the $E E$ stratum.
} 
wages tend to occur for lower and middle quantiles of the distribution. This is the case even when looking at groups with smaller sample sizes. Furthermore, the demographic groups analyzed seem to experience different $Q T E$, both across quantiles and groups. Blacks appear to have larger positive effects at lower quantiles, while Whites appear to have larger effects in the upper quantiles. Also, Non-Hispanic Females show more uninformative results than Non-Hispanic Males. Next, we add Assumption D (stochastic dominance) to tighten these bounds. ${ }^{19}$

\subsection{Bounds on Quantile Treatment Effects Adding Stochastic Dominance}

Estimated bounds for QTE under Assumptions A, B, and D are summarized in Figure 2. The first noteworthy feature of these estimated bounds is that all of them exclude zero at all quantiles for all groups, which strongly suggests that the effect of JC on wages is positive along the wage distribution for all groups. These bounds speak to the identifying power of the stochastic dominance assumption (Assumption D). Also noteworthy is that the general conclusions drawn from the estimated bounds in the previous subsection are maintained and reinforced in several instances.

Looking at the results for the full sample and Non-Hispanics (Figures 2(a) and 2(b)), we again see a shift toward more positive effects when Hispanics are dropped. Interestingly, in both of these samples, the lower and upper bounds for the quantiles 0.55 and 0.8 coincide, resulting in a point-identified effect of JC on wages for these two quantiles. Also, adding the stochastic dominance assumption results in 95 percent IM confidence intervals that exclude zero for most of the quantiles except for 0.05, 0.1, 0.6, 0.9, and 0.95 for the full sample and $0.1,0.25$, and 0.35 for the Non-Hispanic sample. Concentrating on the latter sample, for which Assumption B is likely satisfied, and excluding the bounds for the quantile 0.05 that differ from the rest, the bounds that exclude zero are between (roughly) 2.7 and 11.7 percent. In addition, the IM confidence intervals that exclude zero largely overlap, suggesting that the effects of JC on wages do not differ substantially

\footnotetext{
${ }^{19} \mathrm{Just}$ as with average effects, it is possible to construct bounds on QTE disposing of the individual monotonicity assumption. While still too wide to rule out zero, several of the differences across groups and quantiles pointed out in this section hold for those bounds. These results are available from the authors upon request.
} 
across quantiles. The only clear outliers are the estimated bounds on the 0.05 quantile, which are between 10.5 and 20 percent. In summary, we take these results as clear indication that JC has a significantly positive effect on wages along the wage distribution under the maintained assumptions.

The results by race are shown in Figures 2(c) and 2(d). Adding Assumption D reinforces the notion that Blacks likely exhibit larger positive impacts of JC on log wages in the lower portion of the wage distribution, while Whites likely exhibit larger impacts on the upper quantiles. Indeed, the 95 percent IM confidence intervals for Blacks in the lowest quantiles exclude zero but not those at the highest quantiles. The opposite is true for Whites. However, despite this evidence being stronger than before, it appears inconclusive when looking at the IM confidence intervals, since there is a considerable amount of overlap on the intervals for both groups within quantiles. The IM confidence intervals also show that Blacks have statistically significant positive effects of JC on wages throughout their wage distribution (except at quantiles 0.1, 0.25, 0.9, and 0.95), with estimated bounds that are between roughly 3.1 and 11.5 percent (excluding the 0.05 quantile). Whites show statistically significant positive effects only for quantiles larger than 0.4 (except 0.8), with estimated bounds that are between roughly 6.1 to 14 percent.

Figures 2(e) and 2(f) present the results by Non-Hispanic gender groups. All the estimated bounds under Assumptions A, B, and D for these groups exclude zero at all quantiles, strongly suggesting positive effects of JC on wages and illustrating the identifying power of adding the stochastic dominance assumption. When taking into consideration the 95 percent IM confidence intervals, we find statistically significant positive effects of JC on log wages for more than half of the quantiles considered. Interestingly, NonHispanic Females do not have any statistically significant effects throughout the lower half of their wage distribution up to quantile 0.4 (except at the 0.2 quantile), suggesting that Non-Hispanic Females in the upper half of the distribution are more likely to benefit from higher wages due to JC training. Aside from this distinction, there does not seem to be other substantial differences between gender groups, as judged by the large overlap in their IM confidence intervals. Considering confidence intervals that exclude zero, Non-Hispanic Females have estimated bounds that are between roughly 4.4 to 12.1 percent, while those estimated bounds for Non-Hispanic Males are between roughly 3.6 
to 13.4 percent (excluding the 0.05 quantile). ${ }^{20}$

\section{Conclusion}

We empirically assess the effect of the Job Corps (JC) training program on wages using data from the National Job Corps Study (NJCS), a randomized evaluation of the program. JC is the United States' largest job training program targeting disadvantaged youth and its stated goal is to enhance participants' human capital and labor market outcomes. Thus, research shedding light on the effects of JC on wages is important because wages can be related to human capital improvements due to the program. Furthermore, assessments of the effectiveness of JC are opportune given recent discussions in the public arena seeking to cut federal spending on training programs. Our results provide substantial evidence that JC has positive and significant effects on wages, not only at the mean but also at different points of the wage distribution, and for different demographic groups of interest.

Our empirical approach makes use of recent partial identification results for treatment effects in the presence of sample selection due to Zhang et al. (2008), Imai (2008), and Lee (2009). This bounding strategy allows us to estimate informative nonparametric bounds on the average and quantile treatment effects of JC on wages accounting for non-random selection into employment under weaker assumptions than those conventionally invoked for point identification. We exploit the random assignment in the NJCS to construct "worst case" bounds (Horowitz and Manski, 2000), and then add an individual-level monotonicity assumption on the effect of JC on employment to tighten them. While these bounds cannot rule out negative average effects of JC on wages for those employed irrespective of treatment assignment, by constructing bounds on quan-

\footnotetext{
${ }^{20}$ Recall that Assumption D (stochastic dominance) is stronger than Assumption C (weak monotonicity of mean potential outcomes). To indirectly gauge the plausibility of Assumption D in a similar fashion as Assumption C (see section 6.3), we proceeded to divide each corresponding sample into quintiles based on a given pre-treatment covariate (we employ the same covariates as in section 6.3). Then, for each quintile we compute and test the difference in the average pre-treatment covariate between the $E E$ and $N E$ strata. As it was the case with Assumption C, we do not find evidence against the stochastic dominance assumption for any of the samples analyzed. Details and the results of this exercise can be found in the Internet Appendix.
} 
tile treatment effects we find that for certain quantiles and demographic groups we are able to statistically rule out zero or negative effects of JC on wages. These results are noteworthy given that the lower bound under these assumptions is likely too pessimistic since it implies a theoretically implausible perfect negative correlation between wages and employment.

To further tighten the above bounds, we add a mean-level weak monotonicity or a stochastic dominance assumption across strata (for average and quantile treatment effects, respectively). This assumption formalizes the notion that individuals in some strata are predicted to have better labor market outcomes than others, hence avoiding the perfect negative correlation between wages and employment implied by the previous assumptions. The estimated bounds for the average effect of JC on wages for the individuals employed irrespective of treatment assignment indicate significant positive effects for all groups analyzed. The estimated bounds for groups that exclude Hispanics are remarkably similar, with an estimated lower bound of about 4.6 percent and an upper bound of about 12 percent. Furthermore, we obtain interesting insights when analyzing bounds on quantile treatment effects for individuals employed irrespective of treatment assignment. In particular, we find that the positive effects of JC on wages largely hold across quantiles but that there are differences across quantiles and demographic groups. The effects for Blacks appear larger in the lower half of their wage distribution, while the effects appear larger for Whites in the upper half of their wage distribution. In addition, Non-Hispanic Females show statistically significant positive effects of JC on wages in the upper part of their wage distribution, but not in the lower part. Our preferred estimated bounds on quantile effects - those imposing individual-level monotonicity and stochastic dominance- for the Non-Hispanic population suggest that the effect of JC on wages across quantiles range from about 2.7 to 11.7 percent. We provide evidence that both of these assumptions are not falsified by the data.

In summary, our results provide evidence of a positive and significant effect of JC on wages for those individuals who would be employed irrespective of treatment assignment. This strongly suggests that the JC program has a positive and significant effect on the human capital of these individuals, and that this investment is rewarded in the labor market in the form of higher wages. These results can be taken as encouraging with regard to the effectiveness of JC, and they provide new insights about how the program 
affects different demographic groups it serves.

\section{References}

Abadie, A., Angrist, J., and Imbens, G. 2002. "Instrumental Variables Estimation of Quantile Treatment Effects." Econometrica, 70: 91-117.

Angrist, J., and Krueger, A. 1999. "Empirical Strategies in Labor Economics." In Orley Ashenfelter and David Card (eds) Handbook of Labor Economics, Volume IIIA, Elsevier.

Angrist, J., and Krueger, A. 2001. "Instrumental Variables and the Search for Identification: From Supply and Demand to Natural Experiments." Journal of Economic Perspectives, 15(4): 69-85.

Blundell, R., Gosling, A., Ichimura, I., and Meghir, C. 2007. "Changes in the Distribution of Male and Female Wages Accounting for Employment Composition Using Bounds." Econometrica 75: 323-363.

Burghardt, J., McConnell, S., Meckstroth, A., Schochet, P., and Homrighausen, J. 1999. "National Job Corps Study: Report on Study Implementation." Mathematica Policy Research, Inc., Princeton, NJ.

Chernozhukov, V. and Hansen, C. 2005. "Notes and Comments an IV Model of Quantile Treatment Effects." Econometrica, 73(1): 245-261.

Flores, C., and Flores-Lagunes, A. 2010. "Nonparametric Partial Identification of Causal Net and Mechanism Average Treatment Effects.", Mimeo, University of Miami.

Flores, C., Flores-Lagunes, A., Gonzales, A., and Neumann, T. Forthcoming. "Estimating the Effects of Length of Exposure to Instruction in a Training Program: The Case of Job Corps." The Review of Economics and Statistics.

Flores-Lagunes, A., Gonzalez, A., and Neumann, T. 2009. "Learning but not Earning? The Impact of Job Corps Training on Hispanic Youth." Economic Inquiry, 48: 651-67.

Frangakis, C., and Rubin, D. 2002. "Principal Stratification in Causal Inference." Biometrics, 58: 21-29.

Frumento, P., Mealli, F., Pacini, B. and Rubin, D. 2011. "Evaluating Causal Effects in the Presence of Noncompliance, Truncation by Death, and Unintended Missing Outcomes." Mimeo, University of Pisa. 
Heckman, J. 1979. "Sample Selection Bias as a Specification Error." Econometrica, 47: $153-162$.

Heckman, J. 1990. "Varieties of Selection Bias." American Economic Review, 80: 313-318.

Heckman, J., LaLonde, R., and Smith, J. 1999. "The Economics and Econometrics of Active Labor Market Programs." In Orley Ashenfelter and David Card (eds.) Handbook of Labor Economics, Volume IIIA, Elsevier.

Heckman, J., and Smith, J. A. 1995. "Assessing the Case for Social Experiments." Journal of Economic Perspectives, 9(2): 85-110.

Heckman, J., and Smith, J. A. 1999. "The Pre-Programme Earnings Dip and the Determinants of Participation in a Social Programme: Implications for Simple Programme Evaluation Strategies." Economic Journal, 109(2): 313-348.

Horowitz, J., and Manski, C. 2000. "Nonparametric Analysis of Randomized Experiments with Missing Covariate and Outcome Data." Journal of the American Statistical Association, 95: 77-84.

Imai, K. 2008. "Sharp Bounds on the Causal Effects in Randomized Experiments with "Truncation-by-Death"." Statistics and Probability Letters, 78: 144-149.

Imbens, G., and Angrist, J. 1994. "Identification and Estimation of Local Average Treatment Effects." Econometrica, 62: 467-476.

Imbens, G., and Manski, C. 2004. "Confidence Intervals for Partially Identified Parameters." Econometrica, 72: 1845-1857.

Imbens, G., and Wooldridge, J. 2009. "Recent Developments in the Econometrics of Program Evaluation." Journal of Economic Literature, 47: 5-86.

Lechner, M., and Melly, B. 2010. "Partial Identification of Wage Effects of Training Programs." Mimeo, University of St. Gallen.

Lee, David S. 2009. "Training Wages, and Sample Selection: Estimating Sharp Bounds on Treatment Effects." Review of Economic Studies, 76: 1071-1102.

Manski, C. 1994. "The Selection Problem." in C. Sims (ed) Advances in Econometrics, Sixth World Congress, vol I, Cambridge, U.K. Cambridge University Press, 143-170.

Manski, C., and Pepper, J. 2000. "Monotone Instrumental Variables: With an Application to the Returns to Schooling." Econometrica, 68: 997-1010.

Schochet, P. 2001. "National Job Corps Study: Methodological Appendixes on the 
Impact Analysis." Mathematica Policy Research, Inc., Princeton, NJ.

Schochet, P., Burghardt, J., and Glazerman, S. 2001. "National Job Corps Study: The Impacts of Job Corps on Participants' Employment and Related Outcomes." Mathematica Policy Research, Inc., Princeton, NJ.

US Department of Labor. 2010. http://www.dol.gov/dol/topic/training/jobcorps.html.

USA Today. 2011. "Training Sprawl Costs U.S. \$18 Billion per Year", February 9, 2011.

van Ours, J. 2004. "The Locking-in Effect of Subsidized Jobs." Journal of Comparative Economics, 32: 37-52.

Zhang, J., and Rubin, D. 2003. "Estimation of Causal Effects via Principal Stratification When Some Outcomes are Truncated by 'Death'." Journal of Educational and Behavioral Statistics, 28: 353-368.

Zhang, J., Rubin, D., and Mealli, F. 2008. "Evaluating the Effect of Job Training Programs on Wages Through Principal Stratification." in D. Millimet et al. (eds) Advances in Econometrics vol XXI, Elsevier.

Zhang, J., Rubin, D., and Mealli, F. 2009. "Likelihood-based Analysis of the Causal Effects of Job Training Programs Using Principal Stratification." Journal of the American Statistical Association, 104: 166-176. 
Table 1. Observed groups based on treatment and employment indicators $\left(T_{i}, S_{i}\right)$ and PS mix within groups.

$\begin{array}{ccc}\text { Groups by observed }\left(T_{i}, S_{i}\right) & \text { PS } & \text { PS (individual monotonicity) } \\ (0,0) & N N \text { and } N E & N N \text { and } N E \\ (1,1) & E E \text { and } N E & E E \text { and } N E \\ (1,0) & N N \text { and } E N & N N \\ (0,1) & E E \text { and } E N & E E\end{array}$

Note: PS stands for principal strata.

Table 2. HM (Horowitz and Manski, 2000) Bounds on average treatment effects for week $208 \ln ($ wage).

\begin{tabular}{|c|c|c|c|}
\hline & Quantity in eq. (5) & $\begin{array}{c}\text { Transformed } \\
\text { wages }\end{array}$ & $\begin{array}{c}\text { Untransformed } \\
\text { wages }\end{array}$ \\
\hline \multicolumn{4}{|l|}{$\begin{array}{l}\text { Bounds on support of } \\
\text { wages }\end{array}$} \\
\hline $5^{\text {th }}$ percentile mean wage & & 2.46 & 4.77 \\
\hline $95^{\text {th }}$ percentile mean wage & & 15.96 & 14.00 \\
\hline $\mathrm{Y}^{\mathrm{LB}}$ & $Y^{L B}$ & 0.90 & -1.55 \\
\hline $\mathrm{Y}^{\mathrm{UB}}$ & $Y^{U B}$ & 2.77 & 5.99 \\
\hline \multicolumn{4}{|l|}{ Control group } \\
\hline Observations & & 3599 & 3599 \\
\hline (i)Employment rate & $\operatorname{Pr}\left(S_{i}=1 \mid T_{i}=0\right)$ & 0.566 & 0.566 \\
\hline (ii)Mean $\ln$ (wage) & $E\left[Y_{i} \mid T_{i}=0, S_{i}=1\right]$ & 1.997 & 1.991 \\
\hline (a)Upper bound & $(i) *(i i)+(1-(i i)) * Y^{U B}$ & 2.332 & 3.729 \\
\hline (b)Lower bound & $(i) *(i i)+(1-(i i)) * Y^{L B}$ & 1.52 & 0.451 \\
\hline \multicolumn{4}{|l|}{ Treatment group } \\
\hline Observations & & 5546 & 5546 \\
\hline (iii)Employment rate & $\operatorname{Pr}\left(S_{i}=1 \mid T_{i}=1\right)$ & 0.607 & 0.607 \\
\hline (iv)Mean ln(wage) & $E\left[Y_{i} \mid T_{i}=1, S_{i}=1\right]$ & 2.031 & 2.028 \\
\hline (c)Upper bound & $(i i i) *(i v)+(1-(i i i)) * Y^{U B}$ & 2.321 & 3.587 \\
\hline (d)Lower bound & $(i i i) *(i v)+(1-(i i i)) * Y^{L B}$ & 1.586 & 0.620 \\
\hline \multicolumn{4}{|l|}{ Treatment Effect } \\
\hline Upper bound & $U B^{H M}$ & 0.802 & 3.135 \\
\hline Lower bound & $L B^{H M}$ & -0.746 & -3.109 \\
\hline Width & $U B^{H M}-L B^{H M}$ & 1.548 & 6.244 \\
\hline
\end{tabular}

Notes: "Transformed wages" are the wages transformed as described in Section 6.1.

Alternatively to using Equation (5) to calculate $U B^{H M}$ and $L B^{H M}$, one may use the upper and lower bounds for the control and treatment group, labeled (a), (b), (c), (d), respectively, and compute: $U B^{H M}=(\mathrm{c})-(\mathrm{b})$ and $L B^{H M}=(\mathrm{d})-(\mathrm{a})$. 
Table 3. Estimated principal strata proportions by demographic groups under analysis.

\begin{tabular}{lcccccc}
\hline & & Non- & & Non- & $\begin{array}{c}\text { Non- } \\
\text { Hispanic } \\
\text { Males }\end{array}$ & $\begin{array}{c}\text { Hispanic } \\
\text { Females }\end{array}$ \\
\hline EE & All & Hispanics & Whites & Blacks & Mal \\
NN & 0.566 & 0.559 & 0.657 & 0.512 & 0.583 & 0.530 \\
NE & 0.393 & 0.392 & 0.303 & 0.436 & 0.377 & 0.410 \\
Observations & 0.041 & 0.049 & 0.040 & 0.052 & 0.040 & 0.060 \\
\hline
\end{tabular}

Note: Non-Hispanics are the full sample (All) minus individuals that reported being Hispanic. All estimates are statistically significant

Table 4. Bounds on the average treatment effect of the $E E$ strata for $\ln$ (wage) in week 208.

\begin{tabular}{|c|c|c|c|c|}
\hline \multirow[b]{2}{*}{ Assumptions: } & \multicolumn{2}{|c|}{ Transformed wages } & \multicolumn{2}{|c|}{ Untransformed wages } \\
\hline & $\mathrm{A}$ and $\mathrm{B}$ & $\mathrm{A}, \mathrm{B}$, and $\mathrm{C}$ & $\mathrm{A}$ and $\mathrm{B}$ & $\mathrm{A}, \mathrm{B}$, and $\mathrm{C}$ \\
\hline \multicolumn{5}{|l|}{ Control group } \\
\hline Number of observations & 3599 & 3599 & 3599 & 3599 \\
\hline (i)Proportion employed & 0.566 & 0.566 & 0.566 & 0.566 \\
\hline $\begin{array}{r}\text { Mean } \ln \text { (wage) for } \\
\text { employed }\end{array}$ & 1.997 & 1.997 & 1.991 & 1.991 \\
\hline \multicolumn{5}{|l|}{ Treatment group } \\
\hline Number of observations & 5546 & 5546 & 5546 & 5546 \\
\hline (ii)Proportion employed & 0.607 & 0.607 & 0.607 & 0.607 \\
\hline $\begin{array}{r}\text { Mean } \ln \text { (wage) for } \\
\text { employed }\end{array}$ & 2.031 & 2.031 & 2.028 & 2.028 \\
\hline $\mathrm{p}=[(\mathrm{ii})-(\mathrm{i})] /(\mathrm{ii})$ & 0.068 & 0.068 & 0.068 & 0.068 \\
\hline $\mathrm{p}^{\text {th }}$ quantile & 1.636 & 1.636 & 1.639 & 1.639 \\
\hline 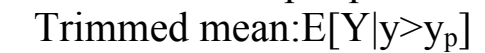 & 2.090 & 2.090 & 2.090 & 2.090 \\
\hline $1-p^{\text {th }}$ quantile & 2.768 & 2.768 & 2.565 & 2.565 \\
\hline Trimmed mean:E[Y $\left.\mid \mathrm{y}<\mathrm{y}_{1-\mathrm{p}}\right]$ & 1.978 & 1.978 & 1.969 & 1.969 \\
\hline \multicolumn{5}{|l|}{ Treatment Effect } \\
\hline Upper bound & $\begin{array}{c}0.093 \\
(0.014)\end{array}$ & $\begin{array}{c}0.093 \\
(0.014)\end{array}$ & $\begin{array}{c}0.099 \\
(0.014)\end{array}$ & $\begin{array}{c}0.099 \\
(0.014)\end{array}$ \\
\hline Lower bound & $\begin{array}{l}-0.019 \\
(0.018)\end{array}$ & $\begin{array}{c}0.034 \\
(0.011)\end{array}$ & $\begin{array}{l}-0.022 \\
(0.016)\end{array}$ & $\begin{array}{c}0.037 \\
(0.012)\end{array}$ \\
\hline Width & 0.112 & 0.059 & 0.121 & 0.062 \\
\hline $\begin{array}{r}95 \text { percent } \\
\text { IM Confidence interval }\end{array}$ & {$[-0.049,0.116]$} & {$[0.016,0.116]$} & {$[-0.048,0.122]$} & {$[0.018,0.122]$} \\
\hline
\end{tabular}

Note: Bootstrap standard errors in parentheses (based on 5,000 replications). IM refers to the Imbens and Manski (2004) confidence interval, which contains the true value of the parameter with a given probability. 
Table 5. Bounds on the average treatment effect of the $E E$ strata for untransformed In(wage) in week 208, by demographic groups.

Panel A: Under Assumptions A and B

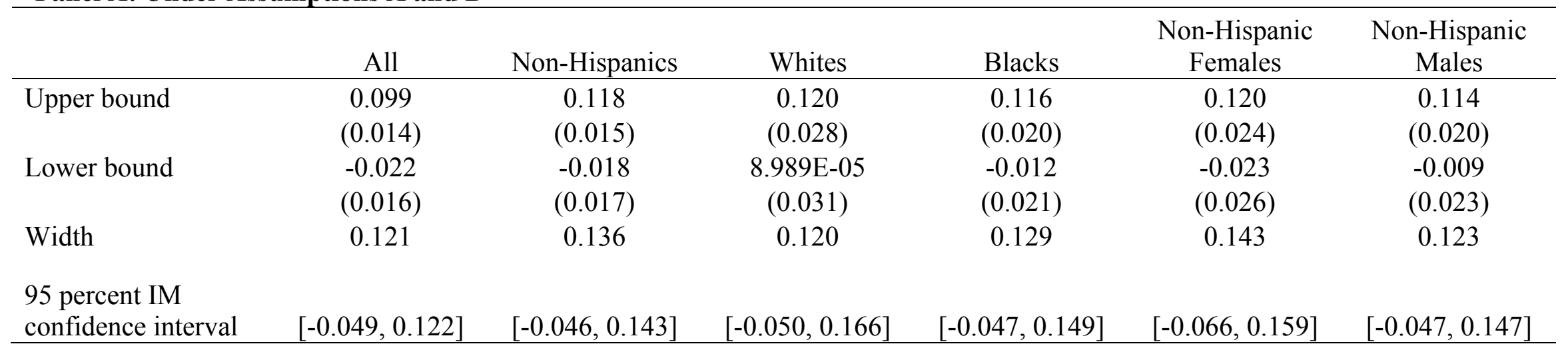

Panel B: Under Assumptions A, B, and C

\begin{tabular}{lcccccc}
\hline & & & & \multicolumn{2}{c}{ Non-Hispanic } & Non-Hispanic \\
& All & Non- Hispanics & Whites & Blacks & Females & 0.120 \\
Upper bound & 0.099 & 0.118 & 0.120 & 0.116 & $(0.020)$ & $(0.024)$ \\
Lower bound & $(0.014)$ & $(0.015)$ & $(0.028)$ & 0.046 & $(0.020)$ \\
& 0.037 & 0.050 & 0.056 & $(0.016)$ & $(0.020)$ & $(0.016)$ \\
Width & $(0.012)$ & $(0.013)$ & $(0.022)$ & 0.063 & 0.074 & 0.061 \\
95 percent IM & 0.062 & 0.068 & 0.064 & & \\
confidence interval & {$[0.018,0.122]$} & {$[0.029,0.143]$} & {$[0.019,0.166]$} & {$[0.027,0.149]$} & {$[0.014,0.159]$} & {$[0.026,0.147]$} \\
\hline
\end{tabular}

Note: Bootstrap standard errors in parentheses (based on 5,000 replications). IM refers to the Imbens and Manski (2004) confidence interval, which contains the true value of the parameter with a given probability. 


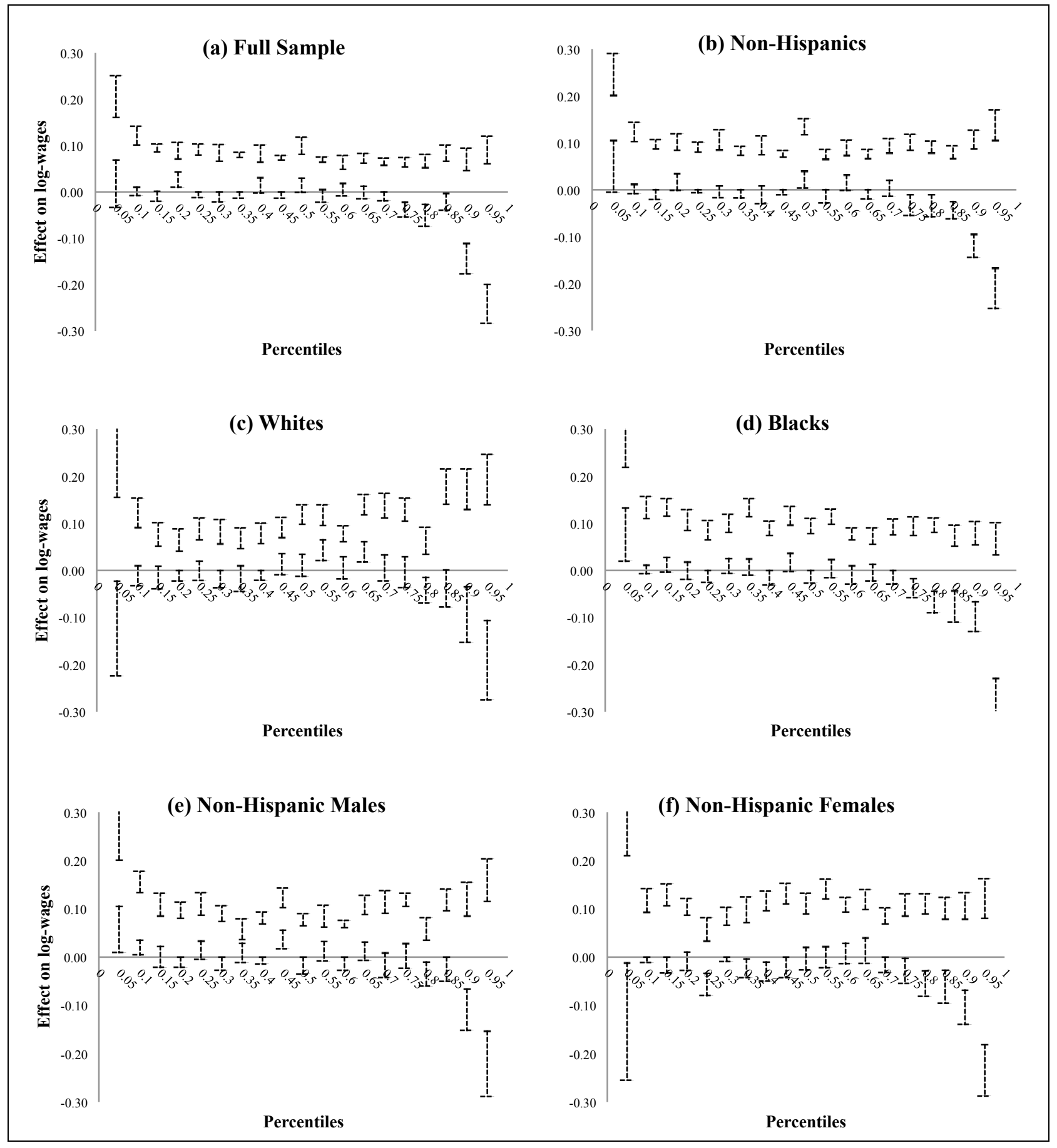

Figure 1. Bounds and 95 percent Imbens and Manski (2004) confidence intervals for $Q T E$ by subgroups, under Assumptions A \& B. Upper and lower bounds are denoted by a short dash, while IM confidence intervals are denoted by a long dash at the end of the dashed vertical lines. 


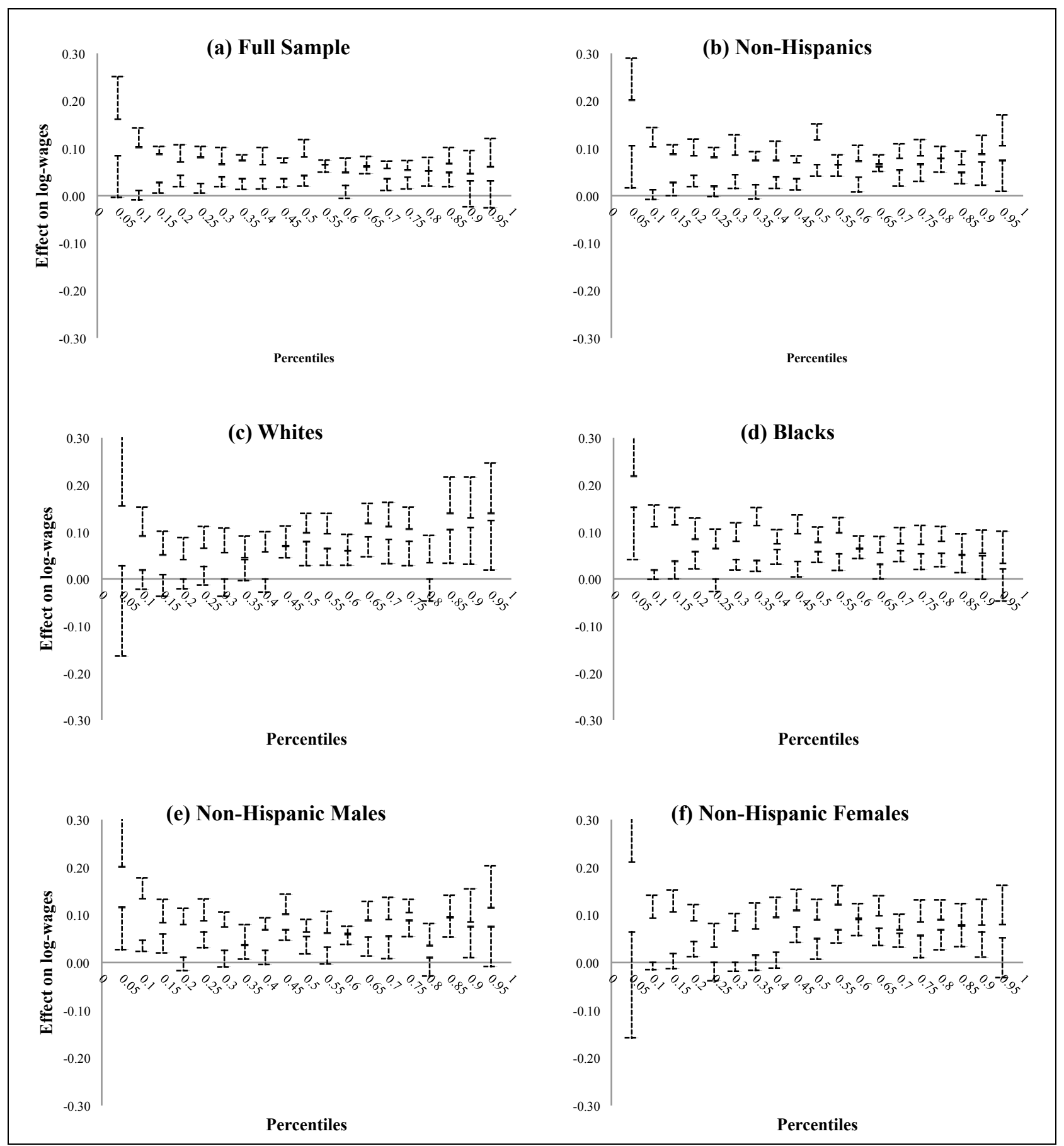

Figure 2. Bounds and 95 percent Imbens and Manski (2004) confidence intervals for $Q T E$ by subgroups, under Assumptions A, B, \& D. Upper and lower bounds are denoted by a short dash, while IM confidence intervals are denoted by a long dash at the end of the dashed vertical lines. 


\title{
INTERNET APPENDIX
}

\section{Bounds on Average and Quantile Treatment Effects of Job Corps Training on Wages}

\author{
German Blanco \\ State University of New York at Binghamton \\ Carlos A. Flores \\ University of Miami \\ Alfonso Flores-Lagunes \\ State University of New York at Binghamton \\ and IZA
}

Discussion Paper No. 6065

October 2011 


\section{Contents}

Tables A1. Summary statistics, by sample

Table A2. Bounds on the average treatment effect of the EE strata for $\ln$ (wage) in week 208, with and without Assumption B.

Table A3. Estimated principal strata proportions by demographic groups

Tables A4. Gauging monotonicity across strata, Assumption C. Indirect evidence by demographic groups

Tables A5. Bounds and confidence intervals on $Q T E_{E E}$ by demographic groups, using Assumptions A and B

Tables A6. Gauging stochastic dominance, Assumption D. Indirect evidence by demographic groups

Tables A7. Bounds and confidence intervals on $Q T E_{E E}$ by demographic groups, using Assumptions A, B and D 


\section{Tables A1. Summary statistics, by sample}

Table A1.1. All sample summary statistics, by treatment status, NJCS.

\begin{tabular}{|c|c|c|c|c|c|c|}
\hline \multirow[t]{2}{*}{ Variable } & \multicolumn{2}{|c|}{ Control } & \multicolumn{2}{|c|}{ Program } & \multicolumn{2}{|c|}{ Difference } \\
\hline & Mean & S.D. & Mean & S.D. & Difference & S.E. \\
\hline Female & 0.458 & 0.498 & 0.452 & 0.498 & -0.006 & 0.010 \\
\hline Age & 18.351 & 2.101 & 18.436 & 2.159 & 0.085 & 0.045 \\
\hline White & 0.263 & 0.440 & 0.266 & 0.442 & 0.002 & 0.009 \\
\hline Black & 0.491 & 0.500 & 0.493 & 0.500 & 0.003 & 0.010 \\
\hline Hispanic & 0.172 & 0.377 & 0.169 & 0.375 & -0.003 & 0.008 \\
\hline Other race & 0.074 & 0.262 & 0.072 & 0.258 & -0.002 & 0.005 \\
\hline Never married & 0.916 & 0.278 & 0.917 & 0.275 & 0.002 & 0.006 \\
\hline Married & 0.023 & 0.150 & 0.020 & 0.139 & -0.003 & 0.003 \\
\hline Living together & 0.040 & 0.197 & 0.039 & 0.193 & -0.002 & 0.004 \\
\hline Separated & 0.021 & 0.144 & 0.024 & 0.154 & 0.003 & 0.003 \\
\hline Has a child & 0.193 & 0.395 & 0.189 & 0.392 & -0.004 & 0.008 \\
\hline \# of children & 0.268 & 0.640 & 0.270 & 0.650 & 0.002 & 0.014 \\
\hline Education & 10.105 & 1.540 & 10.114 & 1.562 & 0.009 & 0.033 \\
\hline Mother's ed. & 11.461 & 2.589 & 11.483 & 2.562 & 0.022 & 0.060 \\
\hline Father's ed. & 11.540 & 2.789 & 11.394 & 2.853 & -0.146 & 0.075 \\
\hline Ever arrested & 0.249 & 0.432 & 0.249 & 0.432 & -0.001 & 0.009 \\
\hline \multicolumn{7}{|l|}{ Household income: } \\
\hline $3,000-6,000$ & 0.208 & 0.406 & 0.206 & 0.405 & -0.002 & 0.011 \\
\hline $6,000-9,000$ & 0.114 & 0.317 & 0.117 & 0.321 & 0.003 & 0.008 \\
\hline $9,000-18,000$ & 0.245 & 0.430 & 0.245 & 0.430 & 0.000 & 0.011 \\
\hline$>18,000$ & 0.182 & 0.386 & 0.179 & 0.383 & -0.003 & 0.010 \\
\hline \multicolumn{7}{|l|}{ Personal income } \\
\hline$<3,000$ & 0.789 & 0.408 & 0.789 & 0.408 & -0.001 & 0.009 \\
\hline $3,000-6,000$ & 0.131 & 0.337 & 0.127 & 0.334 & -0.003 & 0.007 \\
\hline $6,000-9,000$ & 0.046 & 0.209 & 0.053 & 0.223 & 0.007 & 0.005 \\
\hline$>9,000$ & 0.034 & 0.181 & 0.031 & 0.174 & -0.003 & 0.004 \\
\hline \multicolumn{7}{|l|}{ At baseline } \\
\hline Have a job & 0.192 & 0.394 & 0.198 & 0.398 & 0.006 & 0.008 \\
\hline Months employed & 3.530 & 4.238 & 3.596 & 4.249 & 0.066 & 0.089 \\
\hline Had a job & 0.627 & 0.484 & 0.635 & 0.482 & 0.007 & 0.010 \\
\hline Earnings & 2810.482 & 4435.616 & 2906.453 & 6401.328 & 95.971 & 118.631 \\
\hline Usual hrs/week & 20.908 & 20.704 & 21.816 & 21.046 & $0.908 *$ & 0.437 \\
\hline Usual weekly earnings & 102.894 & 116.465 & 110.993 & 350.613 & 8.099 & 5.423 \\
\hline \multicolumn{7}{|l|}{ After random assignment } \\
\hline Week 52 weekly hrs. & 17.784 & 23.392 & 15.297 & 22.680 & $-2.487 *$ & 0.482 \\
\hline Week 104 weekly hrs. & 21.977 & 26.080 & 22.645 & 26.252 & 0.668 & 0.547 \\
\hline Week 156 weekly hrs. & 23.881 & 26.151 & 25.879 & 26.574 & $1.997 *$ & 0.551 \\
\hline Week 208 weekly hrs. & 25.833 & 26.250 & 27.786 & 25.745 & $1.953^{*}$ & 0.544 \\
\hline Week 52 weekly earnings & 103.801 & 159.893 & 91.552 & 149.282 & $-12.249^{*}$ & 3.238 \\
\hline Week 104 weekly earnings & 150.407 & 210.241 & 157.423 & 200.266 & 7.015 & 4.297 \\
\hline Week 156 weekly earnings & 180.875 & 224.426 & 203.714 & 239.802 & $22.839 *$ & 4.855 \\
\hline Week 208 weekly earnings & 200.500 & 230.661 & 227.912 & 250.222 & $27.412 *$ & 5.127 \\
\hline Sample size & 3599 & & 5546 & & & \\
\hline
\end{tabular}

Notes: Missing values for each pretreatment characteristic were imputed with the mean of that variable. Calculations used design weights.

* Indicates that the difference is statistically significant at a 5\% level. 
Table A1.2. Non-Hispanics sample summary statistics, by treatment status, NJCS.

\begin{tabular}{|c|c|c|c|c|c|c|}
\hline \multirow[t]{2}{*}{ Variable } & \multicolumn{2}{|c|}{ Control } & \multicolumn{2}{|c|}{ Program } & \multicolumn{2}{|c|}{ Difference } \\
\hline & Mean & S.D. & Mean & S.D. & Difference & S.E. \\
\hline Female & 0.454 & 0.498 & 0.442 & 0.497 & -0.013 & 0.011 \\
\hline Age & 18.783 & 2.144 & 18.892 & 2.169 & $0.109 *$ & 0.050 \\
\hline White & 0.318 & 0.466 & 0.320 & 0.466 & 0.002 & 0.011 \\
\hline Black & 0.593 & 0.491 & 0.594 & 0.491 & 0.001 & 0.011 \\
\hline Other race & 0.089 & 0.285 & 0.086 & 0.281 & -0.003 & 0.007 \\
\hline Never married & 0.924 & 0.262 & 0.926 & 0.259 & 0.002 & 0.006 \\
\hline Married & 0.018 & 0.132 & 0.015 & 0.122 & -0.003 & 0.003 \\
\hline Living together & 0.037 & 0.187 & 0.036 & 0.183 & -0.002 & 0.004 \\
\hline Separated & 0.020 & 0.140 & 0.023 & 0.149 & 0.003 & 0.003 \\
\hline Has a child & 0.189 & 0.386 & 0.187 & 0.386 & -0.003 & 0.009 \\
\hline \# of children & 0.276 & 0.648 & 0.273 & 0.650 & -0.003 & 0.015 \\
\hline Education & 10.115 & 1.496 & 10.132 & 1.538 & 0.017 & 0.035 \\
\hline Mother's ed. & 11.761 & 1.914 & 11.744 & 1.958 & -0.017 & 0.044 \\
\hline Father's ed. & 11.702 & 1.874 & 11.615 & 1.928 & $-0.088 *$ & 0.044 \\
\hline Ever arrested & 0.257 & 0.433 & 0.256 & 0.433 & -0.001 & 0.010 \\
\hline \multicolumn{6}{|l|}{ Household income: } & 0.008 \\
\hline $3,000-6,000$ & 0.212 & 0.330 & 0.205 & 0.320 & -0.007 & 0.007 \\
\hline $6,000-9,000$ & 0.109 & 0.247 & 0.118 & 0.258 & 0.009 & 0.006 \\
\hline $9,000-18,000$ & 0.246 & 0.348 & 0.243 & 0.342 & -0.003 & 0.008 \\
\hline$>18,000$ & 0.186 & 0.317 & 0.184 & 0.311 & -0.003 & 0.007 \\
\hline \multicolumn{7}{|l|}{ Personal income } \\
\hline$<3,000$ & 0.788 & 0.393 & 0.788 & 0.392 & 0.000 & 0.009 \\
\hline $3,000-6,000$ & 0.136 & 0.330 & 0.129 & 0.322 & -0.007 & 0.007 \\
\hline $6,000-9,000$ & 0.043 & 0.195 & 0.052 & 0.213 & 0.008 & 0.005 \\
\hline$>9,000$ & 0.033 & 0.171 & 0.031 & 0.167 & -0.001 & 0.004 \\
\hline \multicolumn{7}{|l|}{ At baseline } \\
\hline Have a job & 0.188 & 0.386 & 0.203 & 0.398 & 0.015 & 0.009 \\
\hline Months employed & 6.024 & 3.026 & 6.033 & 3.060 & 0.009 & 0.070 \\
\hline Had a job & 0.627 & 0.479 & 0.641 & 0.476 & 0.014 & 0.011 \\
\hline Earnings & 2799.802 & 4221.407 & 2895.404 & 4202.122 & 95.602 & 96.814 \\
\hline Usual hrs/week & 34.773 & 12.010 & 35.376 & 12.088 & $0.602 *$ & 0.277 \\
\hline Usual weekly earnings & 106.572 & 114.224 & 111.672 & 121.653 & 5.100 & 2.711 \\
\hline \multicolumn{7}{|l|}{ After random assignment } \\
\hline Week 52 weekly hrs. & 17.955 & 23.529 & 15.724 & 23.021 & $-2.231 *$ & 0.535 \\
\hline Week 104 weekly hrs. & 21.955 & 26.468 & 22.739 & 26.482 & 0.785 & 0.609 \\
\hline Week 156 weekly hrs. & 23.574 & 26.172 & 26.012 & 26.852 & $2.438^{*}$ & 0.609 \\
\hline Week 208 weekly hrs. & 25.529 & 26.294 & 28.003 & 25.959 & $2.473 *$ & 0.601 \\
\hline Week 52 weekly earnings & 104.211 & 162.416 & 94.053 & 151.607 & $-10.158 *$ & 3.613 \\
\hline Week 104 weekly earnings & 149.629 & 214.956 & 157.384 & 198.883 & 7.754 & 4.763 \\
\hline Week 156 weekly earnings & 176.821 & 222.243 & 204.239 & 241.547 & $27.418^{*}$ & 5.331 \\
\hline Week 208 weekly earnings & 194.657 & 224.183 & 229.703 & 251.732 & $35.046^{*}$ & 5.473 \\
\hline Sample size & 2978 & & 4595 & & & \\
\hline
\end{tabular}

Notes: Same as in Table A1.1. 
Table A1.3. Whites sample summary statistics, by treatment status, NJCS.

\begin{tabular}{|c|c|c|c|c|c|c|}
\hline \multirow[t]{2}{*}{ Variable } & \multicolumn{2}{|c|}{ Control } & \multicolumn{2}{|c|}{ Program } & \multicolumn{2}{|c|}{ Difference } \\
\hline & Mean & S.D. & Mean & S.D. & Difference & S.E. \\
\hline Female & 0.389 & 0.488 & 0.375 & 0.484 & -0.014 & 0.020 \\
\hline Age & 18.860 & 2.186 & 18.889 & 2.132 & 0.029 & 0.089 \\
\hline Never married & 0.878 & 0.325 & 0.887 & 0.315 & 0.010 & 0.013 \\
\hline Married & 0.032 & 0.174 & 0.024 & 0.153 & -0.007 & 0.007 \\
\hline Living together & 0.048 & 0.212 & 0.055 & 0.226 & 0.007 & 0.009 \\
\hline Separated & 0.043 & 0.201 & 0.033 & 0.179 & -0.009 & 0.008 \\
\hline Has a child & 0.124 & 0.323 & 0.100 & 0.296 & -0.024 & 0.013 \\
\hline \# of children & 0.170 & 0.491 & 0.132 & 0.435 & $-0.038 *$ & 0.019 \\
\hline Education & 10.137 & 1.501 & 10.141 & 1.546 & 0.004 & 0.063 \\
\hline Mother's ed. & 11.780 & 1.897 & 11.818 & 1.933 & 0.037 & 0.079 \\
\hline Father's ed. & 11.863 & 1.983 & 11.699 & 2.094 & -0.164 & 0.084 \\
\hline Ever arrested & 0.292 & 0.451 & 0.308 & 0.459 & 0.016 & 0.019 \\
\hline \multicolumn{2}{|l|}{ Household income: } & 0.299 & 0.192 & 0.303 & 0.013 & 0.012 \\
\hline $3,000-6,000$ & 0.173 & 0.305 & 0.177 & 0.300 & 0.004 & 0.012 \\
\hline $6,000-9,000$ & 0.108 & 0.255 & 0.105 & 0.245 & -0.002 & 0.010 \\
\hline $9,000-18,000$ & 0.281 & 0.382 & 0.271 & 0.369 & -0.010 & 0.015 \\
\hline$>18,000$ & 0.260 & 0.385 & 0.256 & 0.376 & -0.005 & 0.016 \\
\hline \multicolumn{7}{|l|}{ Personal income } \\
\hline$<3,000$ & 0.719 & 0.440 & 0.729 & 0.435 & 0.010 & 0.018 \\
\hline $3,000-6,000$ & 0.183 & 0.380 & 0.157 & 0.356 & -0.026 & 0.015 \\
\hline $6,000-9,000$ & 0.059 & 0.231 & 0.071 & 0.253 & 0.012 & 0.010 \\
\hline$>9,000$ & 0.039 & 0.188 & 0.042 & 0.197 & 0.004 & 0.008 \\
\hline \multicolumn{7}{|l|}{ At baseline } \\
\hline Have a job & 0.241 & 0.423 & 0.265 & 0.438 & 0.024 & 0.018 \\
\hline Months employed & 6.395 & 3.372 & 6.410 & 3.298 & 0.015 & 0.137 \\
\hline Had a job & 0.736 & 0.436 & 0.749 & 0.431 & 0.013 & 0.018 \\
\hline Earnings & 3676.064 & 4951.978 & 3870.846 & 4879.621 & 194.782 & 202.509 \\
\hline Usual hrs/week & 34.632 & 13.355 & 36.228 & 14.280 & $1.596^{*}$ & 0.569 \\
\hline Usual weekly earnings & 124.317 & 113.573 & 139.239 & 138.750 & $14.922 *$ & 5.215 \\
\hline \multicolumn{7}{|l|}{ After random assignment } \\
\hline Week 52 weekly hrs. & 22.764 & 24.598 & 20.812 & 25.274 & -1.952 & 1.027 \\
\hline Week 104 weekly hrs. & 26.963 & 27.864 & 28.392 & 28.245 & 1.428 & 1.155 \\
\hline Week 156 weekly hrs. & 28.682 & 26.984 & 30.754 & 28.092 & 2.071 & 1.134 \\
\hline Week 208 weekly hrs. & 30.266 & 25.896 & 33.268 & 26.733 & $3.002 *$ & 1.084 \\
\hline Week 52 weekly earnings & 134.303 & 169.735 & 129.586 & 176.833 & -4.717 & 7.137 \\
\hline Week 104 weekly earnings & 187.490 & 224.168 & 207.121 & 231.743 & $19.631^{*}$ & 9.389 \\
\hline Week 156 weekly earnings & 220.483 & 235.227 & 254.313 & 272.943 & $33.830 *$ & 10.484 \\
\hline Week 208 weekly earnings & 237.449 & 229.065 & 284.036 & 275.967 & $46.587 *$ & 10.432 \\
\hline Sample size & 927 & & 1431 & & & \\
\hline
\end{tabular}

Notes: Same as in Table A1.1. 
Table A1.4. Blacks sample summary statistics, by treatment status, NJCS.

\begin{tabular}{|c|c|c|c|c|c|c|}
\hline \multirow[t]{2}{*}{ Variable } & \multicolumn{2}{|c|}{ Control } & \multicolumn{2}{|c|}{ Program } & \multicolumn{2}{|c|}{ Difference } \\
\hline & Mean & S.D. & Mean & S.D. & Difference & S.E. \\
\hline Female & 0.489 & 0.500 & 0.483 & 0.500 & -0.007 & 0.015 \\
\hline Age & 18.711 & 2.118 & 18.849 & 2.189 & $0.137 *$ & 0.064 \\
\hline Never married & 0.951 & 0.213 & 0.949 & 0.217 & -0.002 & 0.006 \\
\hline Married & 0.010 & 0.098 & 0.010 & 0.099 & 0.000 & 0.003 \\
\hline Living together & 0.028 & 0.163 & 0.023 & 0.149 & -0.005 & 0.005 \\
\hline Separated & 0.011 & 0.102 & 0.017 & 0.129 & 0.006 & 0.003 \\
\hline Has a child & 0.232 & 0.418 & 0.241 & 0.423 & 0.009 & 0.012 \\
\hline \# of children & 0.345 & 0.726 & 0.358 & 0.739 & 0.013 & 0.022 \\
\hline Education & 10.103 & 1.477 & 10.136 & 1.505 & 0.033 & 0.044 \\
\hline Mother's ed. & 11.820 & 1.751 & 11.784 & 1.817 & -0.036 & 0.053 \\
\hline Father's ed. & 11.629 & 1.680 & 11.601 & 1.730 & -0.028 & 0.050 \\
\hline Ever arrested & 0.235 & 0.420 & 0.228 & 0.416 & -0.007 & 0.012 \\
\hline \multicolumn{7}{|l|}{ Household income: } \\
\hline$<3,000$ & 0.285 & 0.363 & 0.282 & 0.361 & -0.003 & 0.011 \\
\hline $3,000-6,000$ & 0.239 & 0.345 & 0.222 & 0.331 & -0.017 & 0.010 \\
\hline $6,000-9,000$ & 0.111 & 0.244 & 0.122 & 0.262 & 0.012 & 0.007 \\
\hline $9,000-18,000$ & 0.221 & 0.320 & 0.228 & 0.326 & 0.007 & 0.010 \\
\hline$>18,000$ & 0.144 & 0.261 & 0.146 & 0.263 & 0.002 & 0.008 \\
\hline \multicolumn{7}{|l|}{ Personal income } \\
\hline$<3,000$ & 0.817 & 0.367 & 0.812 & 0.370 & -0.005 & 0.011 \\
\hline $3,000-6,000$ & 0.117 & 0.306 & 0.121 & 0.310 & 0.004 & 0.009 \\
\hline $6,000-9,000$ & 0.034 & 0.171 & 0.040 & 0.185 & 0.006 & 0.005 \\
\hline$>9,000$ & 0.031 & 0.166 & 0.027 & 0.152 & -0.005 & 0.005 \\
\hline \multicolumn{7}{|l|}{ At baseline } \\
\hline Have a job & 0.162 & 0.364 & 0.170 & 0.371 & 0.008 & 0.011 \\
\hline Months employed & 5.875 & 2.852 & 5.872 & 2.927 & -0.003 & 0.086 \\
\hline Had a job & 0.581 & 0.489 & 0.588 & 0.488 & 0.007 & 0.014 \\
\hline Earnings & 2396.421 & 3667.782 & 2442.836 & 3769.590 & 46.415 & 110.065 \\
\hline Usual hrs/week & 34.765 & 11.298 & 34.821 & 10.623 & 0.056 & 0.325 \\
\hline Usual weekly earnings & 97.175 & 107.057 & 97.378 & 108.423 & 0.203 & 3.189 \\
\hline \multicolumn{7}{|l|}{ After random assignment } \\
\hline Week 52 weekly hrs. & 15.405 & 22.471 & 13.325 & 21.338 & $-2.080 *$ & 0.649 \\
\hline Week 104 weekly hrs. & 19.240 & 24.913 & 20.196 & 25.150 & 0.956 & 0.741 \\
\hline Week 156 weekly hrs. & 20.834 & 24.907 & 23.612 & 25.838 & $2.778^{*}$ & 0.751 \\
\hline Week 208 weekly hrs. & 23.231 & 26.235 & 25.407 & 25.171 & $2.176^{*}$ & 0.761 \\
\hline Week 52 weekly earnings & 86.813 & 150.780 & 76.550 & 132.795 & $-10.263 *$ & 4.210 \\
\hline Week 104 weekly earnings & 129.126 & 208.696 & 132.660 & 173.294 & 3.534 & 5.686 \\
\hline Week 156 weekly earnings & 152.496 & 208.031 & 176.965 & 217.239 & $24.469 *$ & 6.294 \\
\hline Week 208 weekly earnings & 173.397 & 218.869 & 201.068 & 231.578 & $27.671^{*}$ & 6.667 \\
\hline Sample size & 1794 & & 2772 & & & \\
\hline
\end{tabular}

Notes: Same as in Table A1.1. 
Table A1.5. Non-Hispanic Males sample summary statistics, by treatment status, NJCS.

\begin{tabular}{|c|c|c|c|c|c|c|}
\hline \multirow[t]{2}{*}{ Variable } & \multicolumn{2}{|c|}{ Control } & \multicolumn{2}{|c|}{ Program } & \multicolumn{2}{|c|}{ Difference } \\
\hline & Mean & S.D. & Mean & S.D. & Difference & S.E. \\
\hline Age & 18.670 & 2.066 & 18.767 & 2.145 & 0.097 & 0.064 \\
\hline White & 0.356 & 0.479 & 0.358 & 0.479 & 0.002 & 0.015 \\
\hline Black & 0.554 & 0.497 & 0.550 & 0.498 & -0.004 & 0.015 \\
\hline Other race & 0.090 & 0.286 & 0.092 & 0.289 & 0.002 & 0.009 \\
\hline Never married & 0.946 & 0.223 & 0.947 & 0.222 & 0.001 & 0.007 \\
\hline Married & 0.010 & 0.097 & 0.011 & 0.101 & 0.001 & 0.003 \\
\hline Living together & 0.033 & 0.176 & 0.027 & 0.161 & -0.006 & 0.005 \\
\hline Separated & 0.011 & 0.104 & 0.016 & 0.122 & 0.004 & 0.003 \\
\hline Has a child & 0.093 & 0.283 & 0.095 & 0.288 & 0.003 & 0.009 \\
\hline \# of children & 0.121 & 0.408 & 0.125 & 0.416 & 0.004 & 0.013 \\
\hline Education & 10.009 & 1.429 & 10.004 & 1.524 & -0.005 & 0.045 \\
\hline Mother's ed. & 11.808 & 1.833 & 11.796 & 1.937 & -0.012 & 0.058 \\
\hline Father's ed. & 11.734 & 1.892 & 11.629 & 1.971 & -0.105 & 0.059 \\
\hline Ever arrested & 0.321 & 0.463 & 0.321 & 0.463 & 0.000 & 0.014 \\
\hline Household income: & \multicolumn{5}{|c|}{ Household income: } & 0.010 \\
\hline $3,000-6,000$ & 0.201 & 0.319 & 0.192 & 0.303 & -0.009 & 0.010 \\
\hline $6,000-9,000$ & 0.107 & 0.244 & 0.114 & 0.249 & 0.007 & 0.008 \\
\hline $9,000-18,000$ & 0.263 & 0.359 & 0.260 & 0.350 & -0.002 & 0.011 \\
\hline$>18,000$ & 0.204 & 0.332 & 0.209 & 0.331 & 0.005 & 0.010 \\
\hline \multicolumn{7}{|l|}{ Personal income } \\
\hline$<3,000$ & 0.786 & 0.393 & 0.783 & 0.395 & -0.003 & 0.012 \\
\hline $3,000-6,000$ & 0.133 & 0.326 & 0.124 & 0.316 & -0.008 & 0.010 \\
\hline $6,000-9,000$ & 0.047 & 0.203 & 0.056 & 0.220 & 0.008 & 0.006 \\
\hline$>9,000$ & 0.034 & 0.174 & 0.037 & 0.182 & 0.003 & 0.005 \\
\hline \multicolumn{7}{|l|}{ At baseline } \\
\hline Have a job & 0.180 & 0.379 & 0.211 & 0.404 & $0.031 *$ & 0.012 \\
\hline Months employed & 6.040 & 3.003 & 6.053 & 3.073 & 0.013 & 0.093 \\
\hline Had a job & 0.632 & 0.478 & 0.654 & 0.471 & 0.022 & 0.015 \\
\hline Earnings & 3002.686 & 4384.264 & 3172.903 & 4449.987 & 170.217 & 135.035 \\
\hline Usual hrs/week & 35.652 & 12.285 & 36.260 & 12.587 & 0.609 & 0.380 \\
\hline Usual weekly earnings & 115.328 & 121.083 & 123.087 & 130.419 & $7.759 *$ & 3.846 \\
\hline \multicolumn{7}{|l|}{ After random assignment } \\
\hline Week 52 weekly hrs. & 19.322 & 24.694 & 17.179 & 24.006 & $-2.143 *$ & 0.745 \\
\hline Week 104 weekly hrs. & 23.953 & 27.723 & 25.381 & 28.219 & 1.428 & 0.855 \\
\hline Week 156 weekly hrs. & 26.087 & 27.791 & 28.265 & 27.829 & $2.178^{*}$ & 0.850 \\
\hline Week 208 weekly hrs. & 28.525 & 28.215 & 30.232 & 27.304 & $1.706^{*}$ & 0.849 \\
\hline Week 52 weekly earnings & 119.890 & 189.953 & 106.517 & 165.697 & $-13.373 *$ & 5.451 \\
\hline Week 104 weekly earnings & 166.555 & 211.962 & 182.464 & 219.703 & $15.909^{*}$ & 6.599 \\
\hline Week 156 weekly earnings & 198.935 & 236.264 & 233.446 & 266.674 & $34.511 *$ & 7.699 \\
\hline Week 208 weekly earnings & 223.342 & 238.795 & 257.811 & 276.422 & $34.469^{*}$ & 7.893 \\
\hline Sample size & 1805 & & 2475 & & & \\
\hline
\end{tabular}

Notes: Same as in Table A1.1. 
Table A1.6. Non-Hispanics Females sample summary statistics, by treatment status, NJCS.

\begin{tabular}{|c|c|c|c|c|c|c|}
\hline \multirow[t]{2}{*}{ Variable } & \multicolumn{2}{|c|}{ Control } & \multicolumn{2}{|c|}{ Program } & \multicolumn{2}{|c|}{ Difference } \\
\hline & Mean & S.D. & Mean & S.D. & Difference & S.E. \\
\hline Age & 18.919 & 2.228 & 19.050 & 2.189 & 0.131 & 0.077 \\
\hline White & 0.272 & 0.445 & 0.272 & 0.445 & -0.001 & 0.016 \\
\hline Black & 0.638 & 0.481 & 0.649 & 0.477 & 0.011 & 0.017 \\
\hline Other race & 0.089 & 0.285 & 0.079 & 0.270 & -0.010 & 0.010 \\
\hline Never married & 0.898 & 0.300 & 0.900 & 0.298 & 0.002 & 0.010 \\
\hline Married & 0.028 & 0.165 & 0.022 & 0.144 & -0.007 & 0.005 \\
\hline Living together & 0.042 & 0.200 & 0.046 & 0.208 & 0.003 & 0.007 \\
\hline Separated & 0.031 & 0.173 & 0.033 & 0.177 & 0.001 & 0.006 \\
\hline Has a child & 0.306 & 0.456 & 0.302 & 0.456 & -0.003 & 0.016 \\
\hline \# of children & 0.461 & 0.813 & 0.459 & 0.823 & -0.003 & 0.029 \\
\hline Education & 10.243 & 1.565 & 10.294 & 1.541 & 0.051 & 0.054 \\
\hline Mother's ed. & 11.705 & 2.005 & 11.679 & 1.981 & -0.027 & 0.070 \\
\hline Father's ed. & 11.664 & 1.851 & 11.596 & 1.874 & -0.068 & 0.065 \\
\hline Ever arrested & 0.180 & 0.379 & 0.173 & 0.375 & -0.007 & 0.013 \\
\hline \multicolumn{7}{|l|}{ Household income: } \\
\hline $3,000-6,000$ & 0.225 & 0.342 & 0.221 & 0.340 & -0.004 & 0.012 \\
\hline $6,000-9,000$ & 0.110 & 0.250 & 0.123 & 0.269 & 0.012 & 0.009 \\
\hline $9,000-18,000$ & 0.226 & 0.333 & 0.222 & 0.331 & -0.004 & 0.012 \\
\hline$>18,000$ & 0.165 & 0.295 & 0.151 & 0.280 & -0.014 & 0.010 \\
\hline \multicolumn{7}{|l|}{ Personal income } \\
\hline$<3,000$ & 0.790 & 0.393 & 0.793 & 0.389 & 0.004 & 0.014 \\
\hline $3,000-6,000$ & 0.140 & 0.336 & 0.135 & 0.329 & -0.005 & 0.012 \\
\hline $6,000-9,000$ & 0.039 & 0.185 & 0.047 & 0.203 & 0.008 & 0.007 \\
\hline$>9,000$ & 0.031 & 0.167 & 0.024 & 0.146 & -0.007 & 0.005 \\
\hline \multicolumn{7}{|l|}{ At baseline } \\
\hline Have a job & 0.197 & 0.394 & 0.193 & 0.391 & -0.004 & 0.014 \\
\hline Months employed & 6.005 & 3.054 & 6.008 & 3.044 & 0.003 & 0.106 \\
\hline Had a job & 0.621 & 0.481 & 0.625 & 0.481 & 0.004 & 0.017 \\
\hline Earnings & 2556.145 & 4005.201 & 2544.794 & 3838.801 & -11.351 & 136.874 \\
\hline Usual hrs/week & 33.719 & 11.589 & 34.258 & 11.331 & 0.540 & 0.400 \\
\hline Usual weekly earnings & 96.056 & 104.479 & 97.249 & 107.897 & 1.193 & 3.701 \\
\hline \multicolumn{7}{|l|}{ After random assignment } \\
\hline Week 52 weekly hrs. & 16.314 & 21.944 & 13.886 & 21.580 & $-2.428 *$ & 0.759 \\
\hline Week 104 weekly hrs. & 19.555 & 24.674 & 19.402 & 23.698 & -0.153 & 0.844 \\
\hline Week 156 weekly hrs. & 20.556 & 23.746 & 23.164 & 25.285 & $2.608 *$ & 0.854 \\
\hline Week 208 weekly hrs. & 21.931 & 23.288 & 25.186 & 23.864 & $3.255^{*}$ & 0.822 \\
\hline Week 52 weekly earnings & 85.382 & 118.711 & 78.306 & 130.009 & -7.075 & 4.335 \\
\hline Week 104 weekly earnings & 129.302 & 216.840 & 125.696 & 163.592 & -3.607 & 6.726 \\
\hline Week 156 weekly earnings & 150.263 & 201.030 & 167.337 & 199.492 & $17.075^{*}$ & 6.984 \\
\hline Week 208 weekly earnings & 160.208 & 199.982 & 194.189 & 211.335 & $33.981^{*}$ & 7.168 \\
\hline Sample size & 1173 & & 2120 & & & \\
\hline
\end{tabular}

Notes: Same as in Table A1.1. 


\section{Table A2. Bounds on the average treatment effect of the EE strata for $\ln$ (wage) in week 208, with and without Assumption B.}

\begin{tabular}{lcccc}
\hline Assumption & A and B & A, B and C & A & A and C \\
\hline Effect & 0.099 & 0.099 & 0.464 & 0.464 \\
Upper bound & $(0.014)$ & $(0.014)$ & $(0.021)$ & $(0.021)$ \\
& -0.022 & 0.037 & -0.430 & -0.371 \\
Lower bound & $(0.016)$ & $(0.012)$ & $(0.025)$ & $(0.021)$ \\
& 0.121 & 0.062 & 0.894 & 0.835 \\
Width & {$[-0.049,0.122]$} & {$[0.018,0.122]$} & {$[-0.471,0.498]$} & {$[-0.406,0.498]$} \\
95 percent IM & \multicolumn{4}{l}{} \\
Confidence interval & \multicolumn{4}{l}{ Note: Effects are on untransformed wages. }
\end{tabular}

\section{Table A3. Estimated principal strata proportions by demographic groups}

\begin{tabular}{|c|c|c|c|c|c|c|c|}
\hline Strata & All & $\begin{array}{c}\text { Non- } \\
\text { Hispanic }\end{array}$ & Whites & Blacks & $\begin{array}{l}\text { NH } \\
\text { Male }\end{array}$ & $\begin{array}{c}\mathrm{NH} \\
\text { Female }\end{array}$ & Hispanic \\
\hline$\overline{\mathrm{EE}}$ & 0.566 & 0.559 & 0.657 & 0.512 & 0.583 & 0.53 & 0.598 \\
\hline t-statistic & 66.07 & 58.79 & 40.69 & 41.79 & 50.42 & 34.7 & 29.49 \\
\hline $\mathrm{NN}$ & 0.393 & 0.392 & 0.303 & 0.436 & 0.377 & 0.41 & 0.399 \\
\hline t-statistic & 59.67 & 54.1 & 24.41 & 45.49 & 38.17 & 37.98 & 24.58 \\
\hline $\mathrm{NE}$ & 0.041 & 0.049 & 0.04 & 0.052 & 0.04 & 0.06 & 0.002 \\
\hline t-statistic & 3.82 & 4.15 & 1.99 & 3.35 & 2.61 & 3.23 & 0.080 \\
\hline Sample size & 9145 & 7573 & 2358 & 4566 & 4280 & 3293 & 1572 \\
\hline
\end{tabular}

Note: NH stands for Non-Hispanic. 


\section{Tables A4. Gauging monotonicity across strata, As- sumption C. Indirect evidence by demographic groups}

Table A4.1. All sample average labor market pre-treatment covariates by strata, and difference across strata to indirectly test Assumption C.

\begin{tabular}{|c|c|c|c|c|c|}
\hline \multirow[b]{2}{*}{ Variable } & \multirow[b]{2}{*}{ NN } & \multicolumn{2}{|l|}{ Strata } & \multicolumn{2}{|c|}{ Difference across } \\
\hline & & $\mathrm{EE}$ & NE & EE vs. NN & EE vs. NE \\
\hline Earnings & 1996.025 & 3399.915 & 3654.362 & $\begin{array}{l}1403.891 \\
(129.004)\end{array}$ & $\begin{array}{c}-254.447 \\
(1297.027)\end{array}$ \\
\hline Had a job & 0.536 & 0.701 & 0.578 & $\begin{array}{c}0.165 \\
(0.015)\end{array}$ & $\begin{array}{c}0.123 \\
(0.121)\end{array}$ \\
\hline Months employed & 5.582 & 6.300 & 6.416 & $\begin{array}{c}0.718 \\
(0.094)\end{array}$ & $\begin{array}{l}-0.115 \\
(0.766)\end{array}$ \\
\hline Education & 9.846 & 10.277 & 10.323 & $\begin{array}{c}0.431 \\
(0.049)\end{array}$ & $\begin{array}{c}-0.046 \\
(0.386)\end{array}$ \\
\hline
\end{tabular}

Notes: Bootstrapped standard errors for the difference across strata are in parenthesis.

Table A4.2. Non-Hispanics sample average labor market pre-treatment covariates by strata, and difference across strata to indirectly test Assumption C.

\begin{tabular}{lccccc}
\hline & \multicolumn{3}{c}{ Strata } & \multicolumn{3}{c}{ Difference across } \\
Variable & $\mathrm{NN}$ & $\mathrm{EE}$ & $\mathrm{NE}$ & EE vs. NN & EE vs. NE \\
\hline Earnings & 2041.192 & 3385.669 & 3142.984 & 1344.477 & 242.685 \\
& & & & $(141.561)$ & $(946.552)$ \\
Had a job & 0.543 & 0.702 & 0.587 & 0.159 & 0.115 \\
& & & & $(0.016)$ & $(0.112)$ \\
Months employed & 5.602 & 6.318 & \multirow{2}{*}{6.142} & 0.716 & 0.175 \\
& & & & $(0.104)$ & $(0.704)$ \\
Education & \multirow{2}{*}{10.289} & \multirow{2}{*}{10.266} & 0.421 & 0.024 \\
& & & & $(0.052)$ & $(0.347)$ \\
\hline
\end{tabular}

Notes: Same as in Table A4.1. 
Table A4.3. Whites sample average labor market pre-treatment covariates by strata, and difference across strata to indirectly test Assumption C.

\begin{tabular}{lccccc}
\hline \multirow{2}{*}{ Variable } & \multicolumn{3}{c}{ Strata } & \multicolumn{3}{c}{ Difference across } \\
& $\mathrm{NN}$ & $\mathrm{EE}$ & $\mathrm{NE}$ & EE vs. NN & EE vs. NE \\
\hline Earnings & 3174.900 & 4220.867 & 946.539 & 1045.967 & 3274.328 \\
& & & & $(318.134)$ & $(2470.000)$ \\
Had a job & 0.697 & 0.783 & 0.423 & 0.086 & 0.360 \\
& & & & $(0.028)$ & $(0.223)$ \\
Months employed & 5.907 & \multirow{2}{*}{6.698} & 5.307 & 0.792 & 1.391 \\
& & & & $(0.211)$ & $(1.691)$ \\
Education & \multirow{2}{*}{10.242} & 10.128 & 0.326 & 0.114 \\
& & & & $(0.095)$ & $(0.779)$ \\
\hline
\end{tabular}

Notes: Same as in Table A4.1.

Table A4.4. Blacks sample average labor market pre-treatment covariates by strata, and difference across strata to indirectly test Assumption C.

\begin{tabular}{lccccc}
\hline \multirow{2}{*}{ Variable } & \multicolumn{3}{c}{ Strata } & \multicolumn{3}{c}{ Difference across } \\
\hline Earnings & 1605.477 & 2932.503 & 4198.609 & 1327.026 & -1266.106 \\
& & & & $(159.152)$ & $(1032.246)$ \\
Had a job & 0.482 & 0.658 & 0.727 & 0.175 & -0.069 \\
& & & & $(0.022)$ & $(0.139)$ \\
Months employed & 5.493 & \multirow{2}{*}{6.129} & \multirow{2}{*}{6.553} & 0.636 & -0.424 \\
& & & & $(0.126)$ & $(0.805)$ \\
Education & \multirow{2}{*}{10.318} & 10.432 & 0.468 & -0.114 \\
& & & & $(0.065)$ & $(0.421)$ \\
\hline
\end{tabular}

Notes: Same as in Table A4.1. 
Table A4.5. Non-Hispanic Males sample average labor market pre-treatment covariates by strata, and difference across strata to indirectly test Assumption $\mathbf{C}$.

\begin{tabular}{lccccc}
\hline \multirow{2}{*}{ Variable } & \multicolumn{3}{c}{ Strata } & \multicolumn{3}{c}{ Difference across } \\
\hline Earnings & 2223.791 & 3618.566 & 3496.857 & 1394.775 & 121.709 \\
& & & & $(188.053)$ & $(1614.536)$ \\
Had a job & 0.544 & 0.707 & 0.643 & 0.163 & 0.064 \\
& & & & $(0.022)$ & $(0.178)$ \\
Months employed & 5.704 & 6.322 & 5.263 & 0.617 & 1.058 \\
& & & & $(0.135)$ & $(1.107)$ \\
Education & \multirow{2}{*}{5.744} & 10.162 & 10.218 & 0.418 & -0.056 \\
& & & & $(0.067)$ & $(0.542)$ \\
\hline
\end{tabular}

Notes: Same as in Table A4.1.

Table A4.6. Non-Hispanic Females sample average labor market pre-treatment covariates by strata, and difference across strata to indirectly test Assumption C.

\begin{tabular}{|c|c|c|c|c|c|}
\hline \multirow[b]{2}{*}{ Variable } & \multicolumn{3}{|c|}{ Strata } & \multicolumn{2}{|c|}{ Difference across } \\
\hline & NN & $\mathrm{EE}$ & $\mathrm{NE}$ & EE vs. NN & EE vs. NE \\
\hline Earnings & 1828.909 & 3077.960 & 2828.645 & $\begin{array}{l}1249.051 \\
(211.355)\end{array}$ & $\begin{array}{c}249.314 \\
(1127.382)\end{array}$ \\
\hline Had a job & 0.542 & 0.694 & 0.539 & $\begin{array}{c}0.152 \\
(0.025)\end{array}$ & $\begin{array}{c}0.156 \\
(0.141)\end{array}$ \\
\hline Months employed & 5.483 & 6.313 & 6.881 & $\begin{array}{c}0.829 \\
(0.164)\end{array}$ & $\begin{array}{l}-0.568 \\
(0.880)\end{array}$ \\
\hline Education & 10.014 & 10.458 & 10.320 & $\begin{array}{c}0.444 \\
(0.080) \\
\end{array}$ & $\begin{array}{c}0.138 \\
(0.459) \\
\end{array}$ \\
\hline
\end{tabular}

Notes: Same as in Table A4.1. 


\section{Tables A5. Bounds and confidence intervals on $Q T E_{E E}$ by demographic groups, using Assumptions A and B}

Table A5.1 Bounds on the quantile treatment effect of the $E E$ strata for untransformed In(wage) in week 208, by demographic groups, using assumptions $A$ and B.

\begin{tabular}{|c|c|c|c|c|c|c|c|c|c|c|c|c|}
\hline \multirow{2}{*}{$\begin{array}{l}\alpha- \\
\text { percent } \\
\text { ile }\end{array}$} & \multicolumn{2}{|c|}{ All } & \multicolumn{2}{|c|}{ Non-Hispanics } & \multicolumn{2}{|c|}{ Whites } & \multicolumn{2}{|c|}{ Blacks } & \multicolumn{2}{|c|}{ NH Males } & \multicolumn{2}{|c|}{ NH Females } \\
\hline & LB & UB & LB & UB & LB & UB & LB & UB & LB & UB & LB & UB \\
\hline 0.05 & 0.068 & 0.161 & 0.105 & 0.201 & -0.02 & 0.155 & 0.133 & 0.218 & 0.105 & 0.201 & -0.01 & 0.210 \\
\hline 0.10 & 0.011 & 0.102 & 0.011 & 0.102 & 0.009 & 0.091 & 0.011 & 0.110 & 0.035 & 0.134 & 0.000 & 0.093 \\
\hline 0.15 & 0.001 & 0.087 & 0.000 & 0.087 & 0.009 & 0.051 & 0.028 & 0.115 & 0.022 & 0.084 & 0.000 & 0.106 \\
\hline 0.20 & 0.043 & 0.071 & 0.034 & 0.083 & 0.000 & 0.041 & 0.018 & 0.085 & 0.000 & 0.080 & 0.010 & 0.087 \\
\hline 0.25 & 0.000 & 0.080 & 0.000 & 0.080 & 0.019 & 0.065 & 0.000 & 0.065 & 0.033 & 0.087 & -0.03 & 0.033 \\
\hline 0.30 & 0.000 & 0.067 & 0.008 & 0.085 & 0.000 & 0.056 & 0.025 & 0.080 & 0.000 & 0.074 & 0.000 & 0.067 \\
\hline 0.35 & 0.000 & 0.074 & 0.000 & 0.074 & 0.009 & 0.046 & 0.024 & 0.113 & 0.029 & 0.036 & -0.00 & 0.071 \\
\hline 0.40 & 0.030 & 0.065 & 0.009 & 0.074 & 0.000 & 0.057 & 0.000 & 0.074 & 0.000 & 0.069 & -0.01 & 0.095 \\
\hline 0.45 & 0.000 & 0.069 & 0.000 & 0.069 & 0.035 & 0.070 & 0.036 & 0.096 & 0.056 & 0.102 & 0.000 & 0.109 \\
\hline 0.50 & 0.030 & 0.081 & 0.039 & 0.117 & 0.034 & 0.098 & 0.000 & 0.078 & 0.000 & 0.065 & 0.020 & 0.089 \\
\hline 0.55 & 0.005 & 0.065 & 0.000 & 0.065 & 0.065 & 0.095 & 0.023 & 0.098 & 0.032 & 0.063 & 0.021 & 0.121 \\
\hline 0.60 & 0.018 & 0.048 & 0.032 & 0.073 & 0.028 & 0.061 & 0.009 & 0.065 & 0.000 & 0.061 & 0.028 & 0.093 \\
\hline 0.65 & 0.012 & 0.062 & 0.000 & 0.066 & 0.061 & 0.118 & 0.013 & 0.055 & 0.031 & 0.088 & 0.039 & 0.098 \\
\hline 0.70 & 0.000 & 0.057 & 0.021 & 0.078 & 0.033 & 0.111 & 0.000 & 0.075 & 0.008 & 0.090 & 0.000 & 0.069 \\
\hline 0.75 & -0.02 & 0.054 & -0.01 & 0.084 & 0.028 & 0.105 & -0.02 & 0.074 & 0.027 & 0.105 & -0.00 & 0.085 \\
\hline 0.80 & -0.03 & 0.051 & -0.01 & 0.078 & -0.02 & 0.034 & -0.04 & 0.080 & -0.01 & 0.035 & -0.03 & 0.090 \\
\hline 0.85 & -0.00 & 0.067 & -0.03 & 0.065 & 0.001 & 0.140 & -0.04 & 0.051 & 0.00 & 0.095 & -0.03 & 0.078 \\
\hline 0.90 & -0.11 & 0.046 & -0.09 & 0.087 & -0.03 & 0.129 & -0.07 & 0.054 & -0.07 & 0.085 & -0.07 & 0.078 \\
\hline 0.95 & -0.20 & 0.061 & -0.18 & 0.105 & -0.10 & 0.139 & -0.23 & 0.033 & -0.15 & 0.115 & -0.18 & 0.080 \\
\hline
\end{tabular}

Note: NH stands for Non-Hispanic; UB and LB stand for upper and lower bound, respectively. 
Table A5.2 95 percent Imbens and Manski (2004) confidence interval for Bounds on Table A5.1.

\begin{tabular}{|c|c|c|c|c|c|c|c|c|c|c|c|c|}
\hline \multirow{2}{*}{$\begin{array}{l}\alpha- \\
\text { percen- } \\
\text { tile }\end{array}$} & \multicolumn{2}{|c|}{ All } & \multicolumn{2}{|c|}{ Non-Hispanics } & \multicolumn{2}{|c|}{ Whites } & \multicolumn{2}{|c|}{ Blacks } & \multicolumn{2}{|c|}{ NH Males } & \multicolumn{2}{|c|}{ NH Females } \\
\hline & LB & JB & LB & UB & LB & $\mathrm{B}$ & LB & UB & LB & $\mathrm{B}$ & LB & $\mathrm{B}$ \\
\hline 0.05 & -0.034 & 51 & $0.00 \mathrm{~J}$ & 0.29 & 0.224 & & 0.019 & 0.070 & 0.010 & 0.306 & -0.255 & 0.398 \\
\hline 0.10 & 008 & 2 & -0.008 & 4 & 033 & 3 & .007 & 57 & 005 & 0. & 11 & 0.142 \\
\hline 0.15 & 020 & 0 & -0 & 0 & -0.039 & & -0.004 & & 021 & & & 0.152 \\
\hline 0.20 & & & & & -0.022 & 0.088 & -0.019 & 0.129 & -0.021 & 0.114 & -0.027 & 0.122 \\
\hline 0.25 & 12 & 0 . & 0. & -2 & 21 & & -0.025 & 0 . & 05 & 0.1 & & 0.082 \\
\hline 0.30 & -0.021 & 0.102 & -0.017 & 28 & -0.037 & & -0.007 & & 27 & 0.106 & -0 & 0.103 \\
\hline 0.35 & -0.014 & 0.086 & -0.018 & 0.093 & -0.045 & 0.091 & -0.010 & 0.152 & -0.011 & 0.080 & -0.043 & 0.124 \\
\hline 0.40 & -0.002 & 0.101 & -0.030 & 15 & -0.021 & 0.1 & -0.031 & 0.105 & -0.014 & 0.094 & -0.050 & 0.137 \\
\hline 0.45 & 014 & 0. & -0.011 & 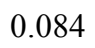 & -0.010 & 0 & -0.002 & 0. & 0.017 & 0.143 & -0 . & 0.153 \\
\hline 0.50 & -0.001 & & 0.0 & & & & -0.027 & & & 0.070 & & 0.132 \\
\hline 0.55 & -0.022 & 0.0 & -0. & 0.086 & 0.021 & & -0.015 & 0 . & -0 . & 0. & -0 & 0.161 \\
\hline 0.60 & -0.009 & 0.07 & -0.001 & 0.106 & -0.018 & 0.0 & -0.030 & 0.0 & -0 . & 0.076 & -0 & 0.123 \\
\hline 0.65 & -0.015 & 0.0 & -0.020 & 0.086 & 0.018 & 0 . & -0.022 & 0.090 & -0 & 28 & -0 & 0.140 \\
\hline 0.70 & & & & & & & & & & & & 0.102 \\
\hline 0.75 & & & & & -0.036 & & -0.058 & & -0.024 & & & 0.132 \\
\hline 0.80 & -0.074 & 0.081 & & & -0.069 & & -0.090 & & -0.060 & 0.081 & -0.081 & 0.131 \\
\hline 0.85 & -0.040 & & -0.062 & 0.094 & -0.078 & & & & -0.050 & & -0.096 & 0.124 \\
\hline 0.90 & -0.177 & 0.09 & -0.145 & 0.127 & -0.153 & 0.2 & -0.130 & 0.104 & -0.152 & 0.155 & -0.139 & 0.133 \\
\hline 0.95 & -0.284 & 0.120 & -0.253 & 0.170 & -0.275 & 0.246 & -0.312 & 0.101 & -0.289 & 0.203 & -0.288 & 0.163 \\
\hline
\end{tabular}

Note: Same as in Table A5.1. 


\section{Tables A6. Gauging stochastic dominance, Assump- tion D. Indirect evidence by demographic groups}

Table A6.1. All sample. Differences in mean labor market pre-treatment covariates across the EE and NE strata (EE-NE), within quintile of the corresponding covariate. This is an indirect test of Assumption D.

\begin{tabular}{lccccc}
\hline Variable & Quintile 1 & Quintile 2 & Quintile 3 & Quintile 4 & Quintile 5 \\
\hline Earnings & 0.00 & -38.06 & 541.57 & -261.28 & -34.27 \\
& $(0.00)$ & $(119.70)$ & $(739.12)$ & $(370.22)$ & $(3510.70)$ \\
Had a job & 0.00 & -0.32 & 0.00 & 0.00 & 0.00 \\
& $(0.00)$ & $(0.22)$ & $(0.00)$ & $(0.00)$ & $(000)$ \\
Months employed & -0.32 & 1.98 & 0.00 & -0.15 & 0.76 \\
& $(0.41)$ & $(1.24)$ & $(0.00)$ & $(0.18)$ & $(0.89)$ \\
Education & 0.83 & -0.01 & $-0.11 *$ & 0.40 & -0.06 \\
& $(0.90)$ & $(0.17)$ & $(0.05)$ & $(0.42)$ & $(0.21)$ \\
\hline
\end{tabular}

Notes: Bootstrapped standard errors for the difference across strata are in parenthesis. See footnote 20 in the paper for details on test implementation.

Table A6.2. Non-Hispanics sample. Differences in mean labor market pre-treatment covariates across the EE and NE strata (EE-NE), within quintile of the corresponding covariate. This is an indirect test of Assumption D.

\begin{tabular}{lccccc}
\hline Variable & Quintile 1 & Quintile 2 & Quintile 3 & Quintile 4 & Quintile 5 \\
\hline Earnings & 0.00 & 16.48 & 524.4 & -222.36 & 2323 \\
& $(0.00)$ & $(36.89)$ & $(606.3)$ & $(399.19)$ & $(2018)$ \\
Had a job & 0.00 & 0.19 & 0.00 & 0.00 & 0.00 \\
& $(0.00)$ & $(0.16)$ & $(0.00)$ & $(0.00)$ & $(0.00)$ \\
Months employed & -0.24 & 0.98 & 0.00 & -0.07 & 0.30 \\
& $(0.29)$ & $(1.10)$ & $(0.00)$ & $(0.12)$ & $(1.25)$ \\
Education & 0.45 & 0.02 & 0.02 & 0.04 & 1.66 \\
& $(0.54)$ & $(0.21)$ & $(0.03)$ & $(0.30)$ & $(1.16)$ \\
\hline
\end{tabular}

Notes: Same as in Table A6.1. 
Table A6.3. Whites sample. Differences in mean labor market pre-treatment covariates across the EE and NE strata (EE-NE), within quintile of the corresponding covariate. This is an indirect test of Assumption D.

\begin{tabular}{lccccc}
\hline Variable & Quintile 1 & Quintile 2 & Quintile 3 & Quintile 4 & Quintile 5 \\
\hline Earnings & 0.00 & 72.77 & -46.38 & 168.53 & -22199 \\
& $(0.00)$ & $(78.58)$ & $(2182)$ & $(373.18)$ & $(23904)$ \\
Had a job & 0.00 & $0.41 *$ & 0.00 & 0.00 & 0.00 \\
& $(0.00)$ & $(0.16)$ & $(0.00)$ & $(0.00)$ & $(0.00)$ \\
Months employed & -2.08 & -1.08 & 0.00 & 0.00 & 15.16 \\
& $(1.02)$ & $(1.97)$ & $(0.00)$ & $(0.22)$ & $(12.54)$ \\
Education & -0.35 & -1.21 & 0.06 & -0.36 & -0.27 \\
& $(0.51)$ & $(2.17)$ & $(0.04)$ & $(1.42)$ & $(0.54)$ \\
\hline
\end{tabular}

Notes: Same as in Table A6.1.

Table A6.4. Blacks sample. Differences in mean labor market pre-treatment covariates across the EE and NE strata (EE-NE), within quintile of the corresponding covariate. This is an indirect test of Assumption D.

\begin{tabular}{lccccc}
\hline Variable & Quintile 1 & Quintile 2 & Quintile 3 & Quintile 4 & Quintile 5 \\
\hline Earnings & 0.00 & 450.1 & -130.9 & -1268.51 & 723.9 \\
& $(0.00)$ & $(511.9)$ & $(283.6)$ & $(829.05)$ & $(1294)$ \\
Had a job & 0.00 & -0.08 & 0.00 & 0.00 & 0.00 \\
& $(0.00)$ & $(0.24)$ & $(0.00)$ & $(0.00)$ & $(0.00)$ \\
Months employed & 0.21 & $-6.56^{*}$ & 0.00 & -0.44 & -1.09 \\
& $(0.28)$ & $(3.12)$ & $(0.00)$ & $(0.32)$ & $(0.91)$ \\
Education & 0.89 & 0.17 & 0.19 & 0.26 & 0.16 \\
& $(0.71)$ & $(0.16)$ & $(0.67)$ & $(0.27)$ & $(0.13)$ \\
\hline
\end{tabular}

Notes: Same as in Table A6.1. 
Table A6.5. Non-Hispanics Males sample. Differences in mean labor market pretreatment covariates across the EE and NE strata (EE-NE), within quintile of the corresponding covariate. This is an indirect test of Assumption D.

\begin{tabular}{lccccc}
\hline Variable & Quintile 1 & Quintile 2 & Quintile 3 & Quintile 4 & Quintile 5 \\
\hline Earnings & 0.00 & -89.64 & 201.8 & -414.97 & 2455 \\
& $(0.00)$ & $(91.71)$ & $(850.7)$ & $(782.10)$ & $(3481)$ \\
Had a job & 0.00 & 0.29 & 0.00 & 0.00 & 0.00 \\
& $(0.00)$ & $(0.68)$ & $(0.00)$ & $(0.00)$ & $(0.00)$ \\
Months employed & -0.38 & -2.69 & 0.00 & -0.29 & 17.37 \\
& $(0.43)$ & $(2.26)$ & $(0.00)$ & $(0.21)$ & $(58.05)$ \\
Education & -0.39 & $0.30^{*}$ & -0.06 & 0.45 & $0.83 *$ \\
& $(0.76)$ & $(0.13)$ & $(0.11)$ & $(0.34)$ & $(0.35)$ \\
\hline
\end{tabular}

Notes: Same as in Table A6.1.

Table A6.6. Non-Hispanics Females sample. Differences in mean labor market pretreatment covariates across the $E E$ and NE strata (EE-NE), within quintile of the corresponding covariate. This is an indirect test of Assumption D.

\begin{tabular}{lccccc}
\hline Variable & Quintile 1 & Quintile 2 & Quintile 3 & Quintile 4 & Quintile 5 \\
\hline Earnings & 0.00 & 61.22 & 423.2 & -151.37 & 2074 \\
& $(0.00)$ & $(66.04)$ & $(810.4)$ & $(510.31)$ & $(2429)$ \\
Had a job & 0.00 & 0.04 & 0.00 & 0.00 & 0.00 \\
& $(0.00)$ & $(0.16)$ & $(0.00)$ & $(0.00)$ & $(0.00)$ \\
Months employed & 0.03 & 4.53 & 0.00 & 0.23 & 0.35 \\
& $(0.46)$ & $(2.03)$ & $(0.00)$ & $(0.17)$ & $(0.89)$ \\
Education & 0.38 & -0.04 & 0.04 & -0.03 & -0.17 \\
& $(0.43)$ & $(0.31)$ & $(0.05)$ & $(0.61)$ & $(0.17)$ \\
\hline
\end{tabular}

Notes: Same as in Table A6.1. 


\section{Tables A7. Bounds and confidence intervals on $Q T E_{E E}$ by demographic groups, using Assumptions A, B and D}

Table A7.1 Bounds on the quantile treatment effect of the $E E$ strata for untransformed In(wage) in week 208, by demographic groups, using assumptions A, B and D.

\begin{tabular}{|c|c|c|c|c|c|c|c|c|c|c|c|c|}
\hline \multirow{2}{*}{$\begin{array}{l}\alpha- \\
\text { percent } \\
\text { ile }\end{array}$} & \multicolumn{2}{|c|}{ All } & \multicolumn{2}{|c|}{ Non-Hispanics } & \multicolumn{2}{|c|}{ Whites } & \multicolumn{2}{|c|}{ Blacks } & \multicolumn{2}{|c|}{ NH Males } & \multicolumn{2}{|c|}{ NH Females } \\
\hline & B & JB & LB & UB & LB & UB & LB & UB & LB & UB & LB & UB \\
\hline 0.05 & 0.084 & & 0 & 0 & 7 & 0 & 53 & 0 & 0.116 & 0.201 & .065 & 0.210 \\
\hline 0.10 & 0.011 & & 0.0 & 0 & 0 & 0 & 0. & & & & .000 & 0.093 \\
\hline 0.15 & 0.027 & 0.087 & 0.027 & 0.087 & 0.009 & 0.051 & 0.038 & 0.115 & 0.060 & 0.084 & 0.019 & 0.106 \\
\hline 0.20 & 0.043 & 071 & 0.043 & 0.083 & 0.000 & 0.041 & 0.058 & 0.085 & 0.012 & 0.080 & 0.044 & 0.087 \\
\hline 0.25 & 0.025 & 080 & 0.020 & 0.080 & 0.026 & 0.0 & 0.000 & & & & 0.000 & 0.033 \\
\hline 0.30 & 0.039 & (1) & 0 & 0.000 & 0.000 & 0.056 & ודם 0. & 0.080 & 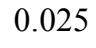 & 0.074 & .000 & 0.067 \\
\hline 0.35 & 0 & 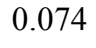 & 0 & 0 & 0.0 & 0. & 0 & 0 & 6 & 0 & 16 & 71 \\
\hline 0.40 & 0.036 & 0.065 & 0.039 & 0.074 & 0.000 & 0.057 & 0.0 & & & & & 0.095 \\
\hline 0.45 & 0.000 & 69 & 0. & 0.069 & 0.069 & 0.070 & 0. & 0.096 & (1) & 0 . & 74 & 0.109 \\
\hline 0.50 & 0042 & 81 & 0 & 0. & 0. & 0 . & 0 & 0 & 0.054 & 0 & 0 & 0.08 \\
\hline 0.55 & 0.065 & 665 & 0.065 & 0.065 & 0.065 & 0.095 & 0.053 & 0.0 & 0.032 & 0.0 & 0.069 & 0.121 \\
\hline 0.60 & 0.022 & 0.048 & 0.038 & 0.073 & 0.061 & 0.061 & 0.065 & 0.065 & 0.058 & 0.061 & 0.090 & 0.093 \\
\hline 0.65 & 0.061 & 62 & 0 . & 0.066 & 0.090 & 0. & 0.0 & 0. & 0 & 0. & 2 & 0.098 \\
\hline 0.70 & & & & & & & 0.061 & & & & 0.061 & 0.069 \\
\hline 0.75 & 0.050 & 0. & 0 & 0. & 0.080 & 0. & 0. & 0 & 0 & 0. & 0.056 & 0.085 \\
\hline 0.80 & 0.051 & 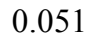 & 0.078 & 0.07 & 0.000 & 0.034 & 0.0 & 0.08 & 010 & 0.0 & 0.069 & 0.090 \\
\hline 0.85 & 0.049 & 0.067 & 0.049 & 0.065 & 0.104 & 0.140 & 0.051 & 0.051 & 0.095 & 0.095 & 0.078 & 0.078 \\
\hline 0.90 & 0.030 & 0.046 & 0.071 & 0.087 & 0.109 & 0.129 & 0.049 & 0.054 & 0.075 & 0.085 & 0.065 & 0.078 \\
\hline 0.95 & 0.031 & 0.061 & 0.074 & 0.105 & 0.124 & 0.139 & 0.021 & 0.033 & 0.076 & 0.115 & 0.052 & 0.080 \\
\hline
\end{tabular}

Note: NH stands for Non-Hispanic; UB and LB stand for upper and lower bound, respectively. 
Table A7.2 95 percent Imbens and Manski (2004) confidence interval for Bounds on Table A7.1.

\begin{tabular}{|c|c|c|c|c|c|c|c|c|c|c|c|c|}
\hline \multirow{2}{*}{$\begin{array}{l}\alpha- \\
\text { percen- } \\
\text { tile }\end{array}$} & \multicolumn{2}{|c|}{ All } & \multicolumn{2}{|c|}{ Non-Hispanics } & \multicolumn{2}{|c|}{ Whites } & \multicolumn{2}{|c|}{ Blacks } & \multicolumn{2}{|c|}{ NH Males } & \multicolumn{2}{|c|}{ NH Females } \\
\hline & LB & UB & LB & UB & LB & UB & LB & UB & LB & UB & LB & UB \\
\hline 0.05 & -0.004 & 0.251 & 0.016 & 0.29 & -0.164 & 0.318 & 0.041 & 0.338 & 0.027 & 0.306 & -0.16 & 0.398 \\
\hline 0.10 & -0.009 & 0.142 & -0.008 & 0.144 & -0.022 & 0.153 & -0.001 & 0.157 & 0.023 & 0.178 & -0.02 & 0.142 \\
\hline 0.15 & 0.005 & 0.104 & 0.000 & 0.107 & -0.037 & 0.101 & 0.000 & 0.152 & 0.020 & 0.132 & -0.01 & 0.152 \\
\hline 0.20 & 0.019 & 0.107 & 0.019 & 0.119 & -0.021 & 0.088 & 0.021 & 0.129 & -0.02 & 0.114 & 0.012 & 0.122 \\
\hline 0.25 & 0.005 & 0.104 & -0.002 & 0.101 & -0.013 & 0.111 & -0.027 & 0.106 & 0.031 & 0.134 & -0.04 & 0.082 \\
\hline 0.30 & 0.019 & 0.102 & 0.015 & 0.128 & -0.037 & 0.108 & 0.019 & 0.119 & -0.01 & 0.106 & -0.02 & 0.103 \\
\hline 0.35 & 0.013 & 0.086 & -0.007 & 0.093 & -0.004 & 0.091 & 0.016 & 0.152 & 0.007 & 0.080 & -0.02 & 0.124 \\
\hline 0.40 & 0.014 & 0.101 & 0.015 & 0.115 & -0.028 & 0.1 & 0.031 & 0.105 & -0.00 & 0.094 & -0.01 & 0.137 \\
\hline 0.45 & 0.018 & 0.079 & 0.012 & 0.084 & 0.045 & 0.113 & 0.004 & 0.136 & 0.047 & 0.143 & 0.042 & 0.153 \\
\hline 0.50 & 0.02 & 0.118 & 0.041 & 0.151 & 0.028 & 0.139 & 0.035 & 0.11 & 0.018 & 0.090 & 0.007 & 0.132 \\
\hline 0.55 & 0.049 & 0.075 & 0.041 & 0.086 & 0.029 & 0.139 & 0.018 & 0.13 & -0.00 & 0.108 & 0.041 & 0.161 \\
\hline 0.60 & -0.006 & 0.079 & 0.008 & 0.106 & 0.029 & 0.095 & 0.043 & 0.091 & 0.038 & 0.076 & 0.057 & 0.123 \\
\hline 0.65 & 0.046 & 0.083 & 0.051 & 0.086 & 0.047 & 0.161 & 0.000 & 0.09 & 0.013 & 0.128 & 0.036 & 0.140 \\
\hline 0.70 & 0.011 & 0.073 & 0.02 & 0.109 & 0.032 & 0.163 & 0.037 & 0.109 & 0.008 & 0.137 & 0.032 & 0.102 \\
\hline 0.75 & 0.014 & 0.074 & 0.03 & 0.118 & 0.028 & 0.153 & 0.020 & 0.114 & 0.054 & 0.132 & 0.010 & 0.132 \\
\hline 0.80 & 0.020 & 0.081 & 0.049 & 0.104 & -0.047 & 0.092 & 0.026 & 0.111 & -0.03 & 0.081 & 0.026 & 0.131 \\
\hline 0.85 & 0.019 & 0.101 & 0.025 & 0.094 & 0.033 & 0.216 & 0.013 & 0.096 & 0.053 & 0.141 & 0.033 & 0.124 \\
\hline 0.90 & -0.024 & 0.095 & 0.022 & 0.127 & 0.031 & 0.216 & -0.001 & 0.104 & 0.010 & 0.155 & 0.011 & 0.133 \\
\hline 0.95 & -0.026 & 0.120 & 0.009 & 0.17 & 0.019 & 0.246 & -0.047 & 0.101 & -0.01 & 0.203 & -0.03 & 0.163 \\
\hline
\end{tabular}

Note: Same as in Table A7.1. 